May 21, 1975

\title{
Parameter Studies for a Two-Component \\ Fusion Experiment
}

\author{
by
}

Harry H. Towner

Graduate Research Assistant

Nuclear Engineering Program.

University of Illinois at Urbana-Champaign

Urbana, Illinois 61801
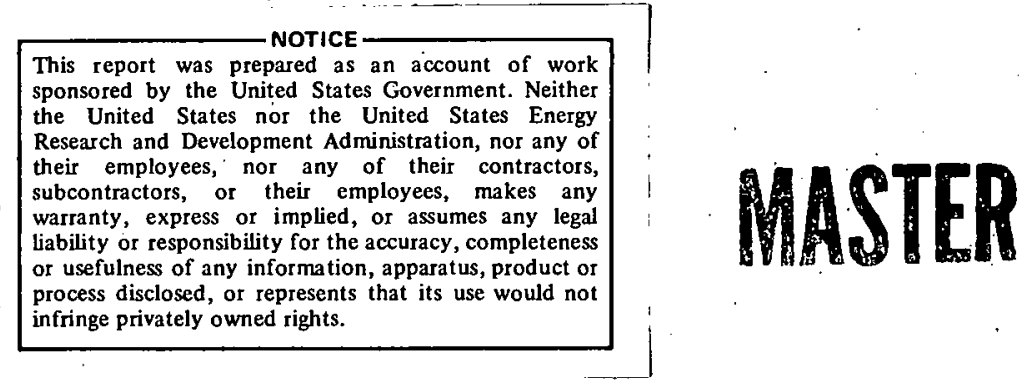

*This work was under the auspices of the U. S. Energy Research and Development Administration under grant AT(11-1) 2218 .

Reports in this series represent research by individual project members. Since the work is frequently incomplete, these memos should not be referenced or distributed further without approval of the Project Coordinator. 


\section{DISCLAIMER}

This report was prepared as an account of work sponsored by an agency of the United States Government. Neither the United States Government nor any agency Thereof, nor any of their employees, makes any warranty, express or implied, or assumes any legal liability or responsibility for the accuracy, completeness, or usefulness of any information, apparatus, product, or process disclosed, or represents that its use would not infringe privately owned rights. Reference herein to any specific commercial product, process, or service by trade name, trademark, manufacturer, or otherwise does not necessarily constitute or imply its endorsement, recommendation, or favoring by the United States Government or any agency thereof. The views and opinions of authors expressed herein do not necessarily state or reflect those of the United States Government or any agency thereof. 


\section{DISCLAIMER}

Portions of this document may be illegible in electronic image products. Images are produced from the best available original document. 


\begin{abstract}
The sensitivity of the energy multiplication of a two-component fusion experiment is examined relative to the following parameters: energy confinement time $\left(\tau_{E}\right)$, particle confinement time $\left(\tau_{p}\right)$, effective $Z$ of the plasma $\left(Z_{\text {eff }}\right)$, injection rate $\left(j_{I}\right)$ and injection energy $\left(E_{I}\right)$. The Energy Research and Development Administration recently approved funding for such a fusion device (the Toroidal Fusion Test Reactor or TFTR) which will be built at the Princeton Plasma Physics Laboratory. Hence, such a parameter study seems both timely and necessary. This work also serves as an independent check on the design values proposed. for the TFTR to enable it to achieve energy breakeven $(F=1)$.

Using the nominal TFTR design parameters and a self-consistent ionelectron power balance, the maximum $F$-value is found to be $\sim 1.2$ which occurs at an injection energy of $\sim 210 \mathrm{KeV}$. The injector operation, i.e. its current and energy capability are shown to be a very critical factor in the TFTR performance. However, if the injectors meet the design objectives, there appears to be sufficient latitude in the other parameters to offer reasonable assurance that energy breakeven can be achieved.
\end{abstract}




\section{ACKNOWLEDGMENTS}

The author wishes to thank his advisor, Professor George H. Miley, without whose contributions this thesis would never have been possible. Appreciation is also expressed to Drs. D. L. Jassby and F. H. Tenney of the Princeton Plasma Physics Laboratory for their technical advice and comments. This work was made possible by the support under AEC contract US AT(11-1)-2218 and Princeton Plasma Physics Laboratory subcontract US AEC Subc.

Finally, this work is dedicated to my wife, Linda, who constantly provided encouragement throughout the course of this work. 
TABLE OF CONTENTS

Chapter

$\underline{\text { Page }}$

INTRODUCTION. . . . . . . . . . . . . . . 1

1 TWO-COMPONENT REACTIVITIES. . . . . . . . . . . 3

Introduction. . . . . . . . . . . . . . . 3

Reactivity Calculation. . . . . . . . . . . 3

Results....................... 5

2 ENERGY MULTIPLICATION FACTORS . . . . . . . . . . 12

Introduction. . . . . . . . . . . . . . ${ }^{1} 12$

F-Value Calculation . . . . . . . . . . 12

G-Value Calculation ................ 17

Results....................... 18

Summary . . . . . . . . . . . . . . . 31

3 POWER BALANCE . . . . . . . . . . . . . . . 35

Introduction. . . . . . . . . . . . . . . 35

The Model . . . . . . . . . . . . . . 35

Method of Solution. . . . . . . . . . . . 43

The Pressure Balance. . . . . . . . . . . . . 46

Results..................... 47

Performance With Design Parameters. . . . . . . . . 48 .

Effect of Parameter Variations. . . . . . . . . 57

4 SUMMARY AND CONCLUSION. . . . . . . . . . . 69

Sensitivity . . . . . . . . . . . . . . 69

Conclusion. ... . . . . . . . . . . . . 72

Recommendation for Future Work. . . . . . . . . . 73

LIST OF REFERENCES. . . . . . . . . . . . . . 75 


\section{INTRODUCTION}

Fusion power has the potential of supplying our world with energy for many millions of years. It has been estimated ${ }^{(1)}$ that with deuterium alone, the total available energy would be at least $10^{21}$ kilowattyears. At the world's present energy consumption rate, this energy. would last for over $10^{9}$ years. However, this vast potential means nothing unless fusion power is both scientifically and economically feasible.

Scientific feasibility is defined in terms of an experiment to demonstrate that as much energy is released from a fusion plasma as is put in. One of the most promising experiments which will hopefully demonstrate scientific feasibility is the Two Component Torus* (TCT). (2) This device is based on the concept of obtaining fusion by high-energy ions colliding with a cold background. While this has been discussed in the literature for some time ${ }^{(3-9)}$, the method recently received a new impetus due to the work of J. M. Dawson, et al. (4) They showed that net thermonuclear power could be achieved under conditions less restrictive than Lawson's criterion. In addition, the cold-target system appears to be quite attractive for use with advanced fuels such as D- ${ }^{3}$ He since both radiation losses and parasitic D-D neutron production are greatly reduced. $(5-8)$

The work presented here consists of three chapters which emphasize reactivities, energy multiplication factors and power balances for twocomponent plasmas. Each of these chapters will present results based

\footnotetext{
* In recent reports this experiment is designated as the Toroidal Fusion Test Reactor (TFTR). Both names (TCT and TFTR) will be used interchangeably throughout the present report.
} 
upon expected TCT parameters ${ }^{(2)}$ for $\mathrm{D} \rightarrow \mathrm{T}$ and for projected advance fuel parameters, mainly $D \rightarrow{ }^{3} \mathrm{He}$. [Here the convention $x \rightarrow y$ indicates that species $x$ is injected into a target plasma of species $y$ and will be used. throughout this work.] 
CHAPTER 1

TWO-COMPONENT REACTIVITIES

\section{Introduction}

....Two-component plasmas will employ injection of highly-energetic .. (beam) ions into a low-temperature (target) plasma.: Since the target ion temperature is low, the majority of the fusion reactions occur between the injected and target ions (vs. target-target or beam-beam ..... reactions). In contrast, fusion reactions in other magnetically-confined thermonuclear plasmas take place between thermalized ions.

\section{Reactivity Calculation}

In general, the reaction rate parameter is defined...as:

$$
\langle\sigma v\rangle=\int_{\vec{v}_{1}} \int_{\vec{v}_{2}} f_{1}\left(\vec{v}_{1}\right) f_{2}\left(\vec{v}_{2}\right)|\vec{v}| \sigma(|\vec{v}|) d \vec{v}_{1} d \vec{v}_{2}
$$

Where $f_{1}\left(\vec{v}_{1}\right)$ and $f_{2}\left(\vec{v}_{2}\right)$ are the velocity distributions of the two fusing species (normalized to unity), $\vec{v}=\vec{v}_{1}-\vec{v}_{2}$ is the relative velocity of the two species, and $\sigma(|\vec{v}|)$ is the fusion cross section for the reactants.

For two-component systems, the velocity distribution for the tar-.. get ions (say species 2) is described by a Maxwellian distribution. That is:

$$
f_{2}\left(\vec{v}_{2}\right)=\frac{\beta^{3}}{\pi^{3 / 2}} \exp \left(-\left(\beta v_{2}\right)^{2}\right) \text {, }
$$

where $\beta^{2}=\frac{m_{2}}{2 T}$. Here the temperature (in energy units) of the background ions is given by $\mathrm{T}$ and their mass as $\mathrm{m}_{2}$. 
The injected-ion velocity distribution can be approximated as a mono-energetic beam, i.e.

$$
f_{1}\left(\vec{v}_{1}\right)=\delta\left(\vec{v}_{1}-\vec{v}_{0}\right)
$$

where $\vec{v}_{0}$ is the velocity of the beam particles.

After changing the integration variables from $\vec{v}_{1}$ and $\vec{v}_{2}$ to $\vec{v}_{1}$ and $\vec{v}$ in $\mathrm{Eq}$. (1.1), the instantaneous two-component reaction-rate parameter or reactivity becomes:

$$
\begin{aligned}
\langle\sigma v\rangle_{b} & =\frac{\beta^{3}}{\pi^{3 / 2}} \int_{\vec{v}_{1}} \delta\left(\vec{v}_{1}-\vec{v}_{0}\right) d \vec{v}_{1} \int_{\vec{v}} \sigma(|\vec{v}|) \cdot|\vec{v}| \\
& x \exp \left(-\beta^{2}\left(v^{2}+v_{1}{ }^{2}+2 \vec{v}_{1} \cdot \vec{v}\right)\right) d \vec{v}
\end{aligned}
$$

Here and in subsequent chapters the subscript $b$ is added to distinguish this reactivity from the normal case for Maxwellian fusion. By using spherical coordinates and letting $\vec{v}_{1}$ lie in the $z$ direction, the integration over $\vec{v}_{1}$ and $\phi$ can be completed and this equation simplifies to:

$$
\begin{aligned}
\langle\sigma v\rangle_{b} & =\frac{2 \beta^{3}}{\sqrt{\pi}} \int_{v=0}^{\infty} \sigma(v) v^{3} \\
& \times \int_{0}^{\pi} \exp \left(-\beta^{2}\left(v^{2}+v_{0}{ }^{2}+2 v v_{0} \cos \theta\right)\right) \sin \theta d \theta d v
\end{aligned}
$$

Finally, performing the $\theta$ integration, the following final form is obtained:

$$
\begin{aligned}
\langle\sigma v\rangle_{b} & =\frac{2 \beta}{\sqrt{\pi}} \frac{1}{v_{0}} \int_{v=0}^{\infty} \sigma(v) v^{2} \exp \left(-\beta^{2}\left(v^{2}+v_{0}^{2}\right)\right) \\
& x \sinh \left(2 \beta^{2} v_{0} v\right) d v .
\end{aligned}
$$




\section{Results}

The numerical evaluation of this equation, using the cross sections in Refs. 10 and 11, is illustrated in Figs. 1.1-1.6.

It is important to note that the $\langle\sigma v\rangle_{b}$ values increase with temperature for injection energies below the peak in the cross section. This trend can be understood physically by observing that in this region the relative ion velocities, on the average, are below the optimum. Hence when the target velocities increase, the relative ion velocities move closer to the optimum, giving a larger $\langle\sigma v\rangle_{b}$ value. Just the reverse effect occurs for energies above the peak.

In the limit where the injection energy is much higher than the target temperature the relative velocity essentially reduces to the velocity of the injected ion. As a result, the $\langle\sigma v\rangle_{b}$ values become temperature dependent for high injection energies.

Finally; it should be noted that the maximum values for the twocomponent reactivities are of the same order of magnitude as that obtained in the usual Maxwellian case. This is encouraging since large power densities are desirable from an economic point of view. Indeed, Jassby ${ }^{(12)}$ has shown that two component systems can potentially achieve quite high power densities. 


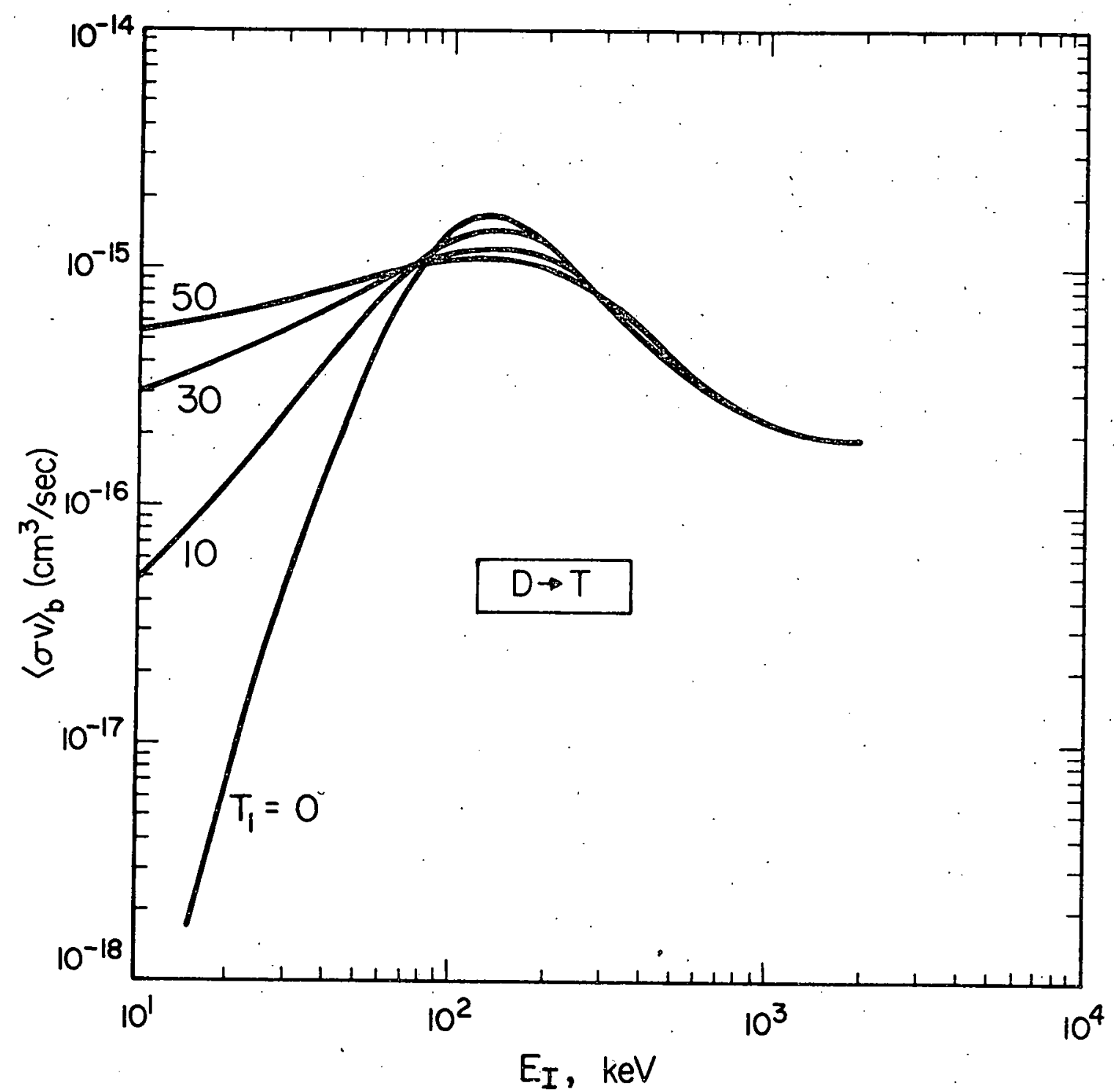

Fig. 1.1 Beam-Maxwellian reactivity for $T(d, n){ }^{4} \mathrm{He}$. 
7

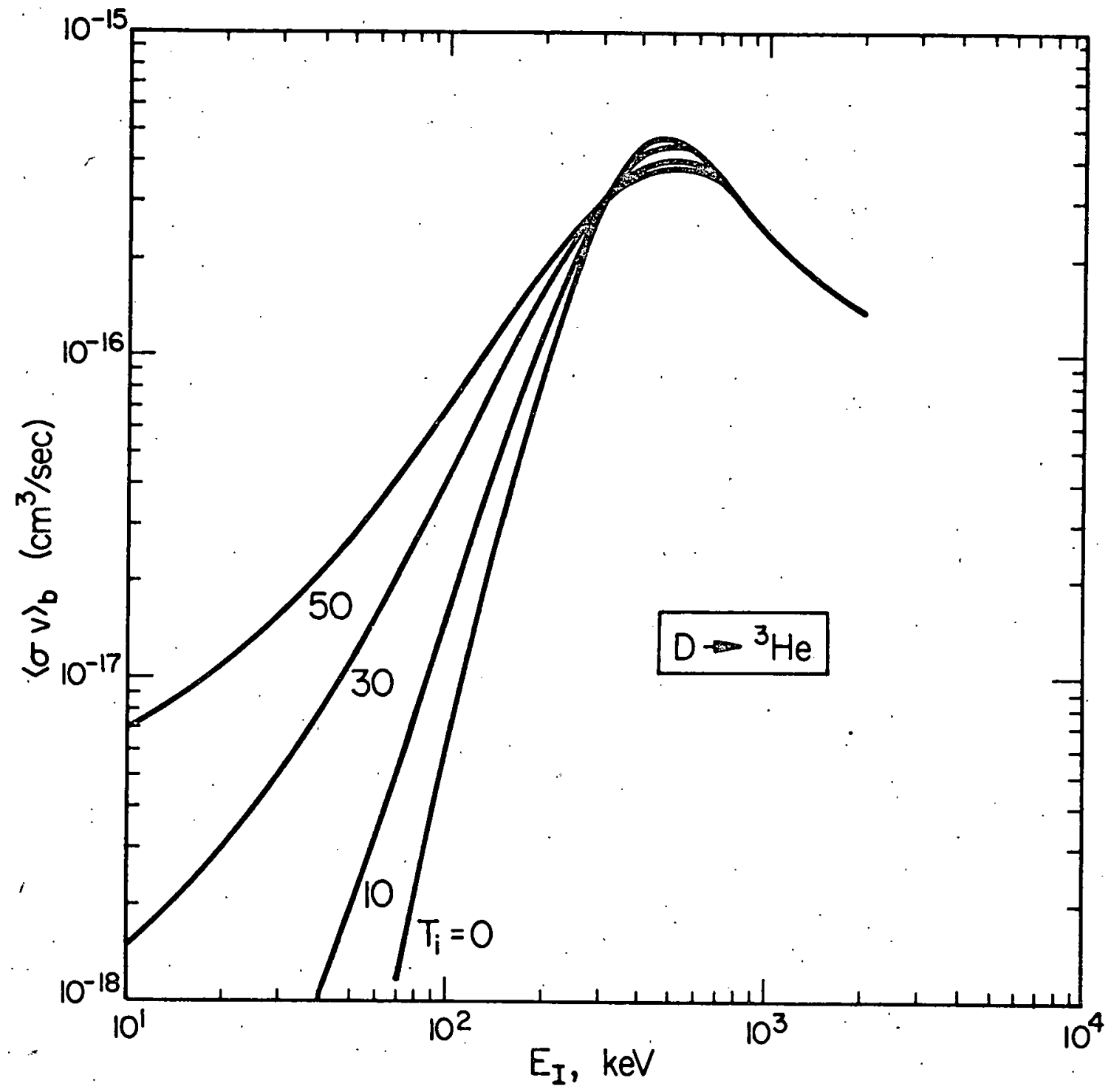

Fig. 1.2 Beam-Maxwellian reactivity for ${ }^{3} \mathrm{He}(\mathrm{d}, \mathrm{p}){ }^{4} \mathrm{He}$. 


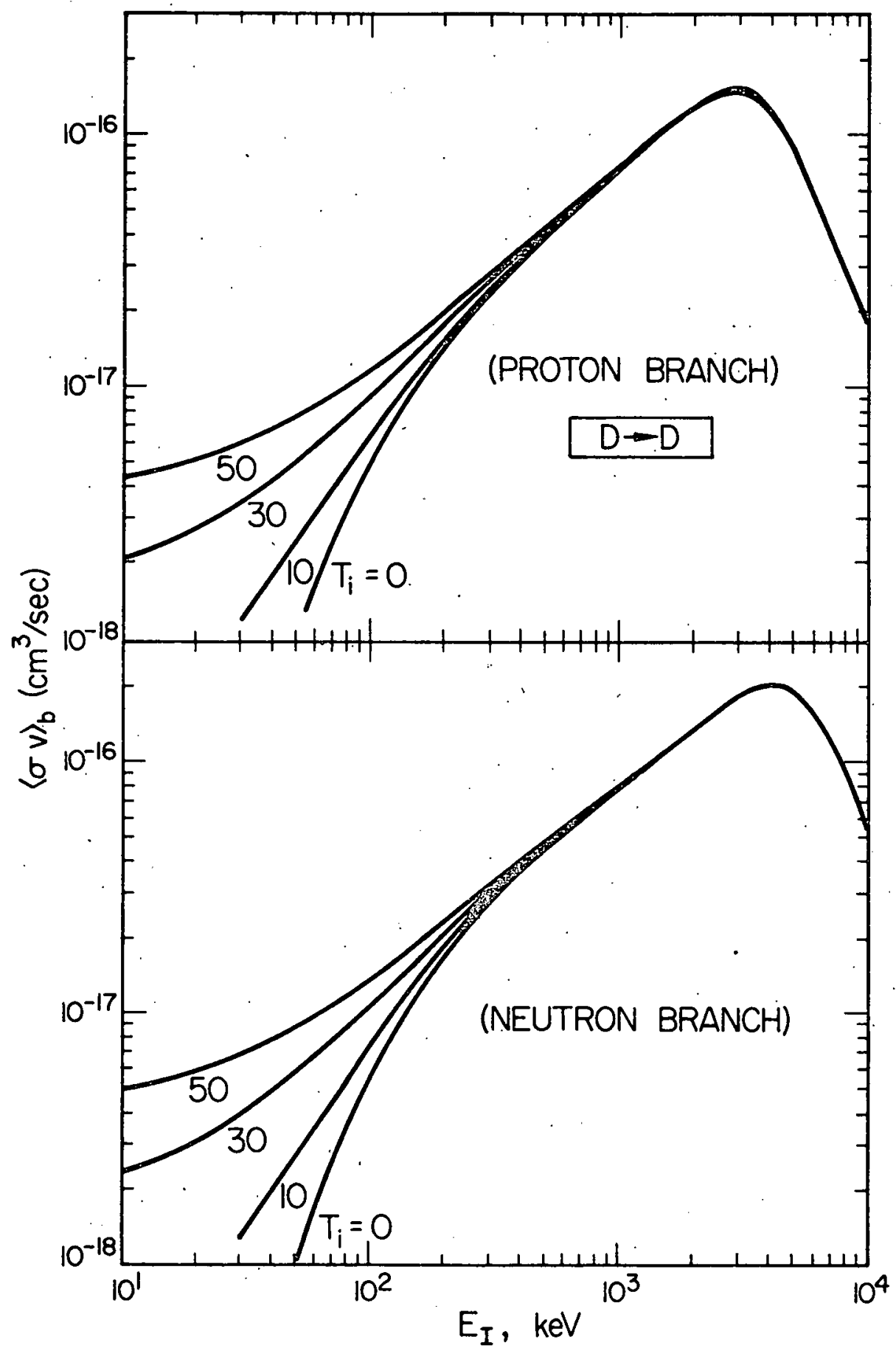

Fig. 1.3 Beam-Maxwellian reactivity for $D(d, p) T$ and $D(d, n){ }^{3} \mathrm{He}$. 


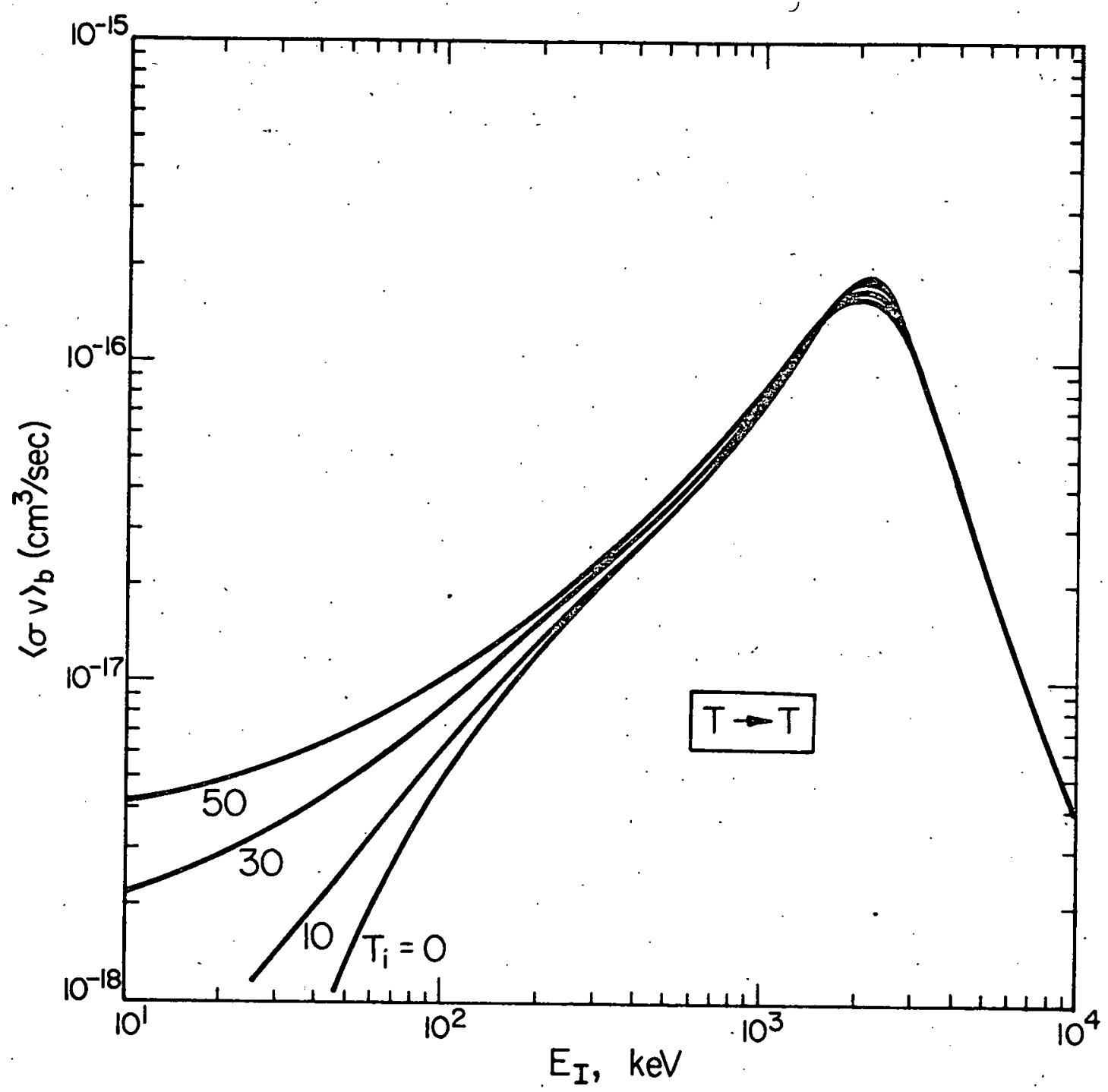

Fig. 1.4 Beam-Maxwellian reactivity for $T(t, 2 n)^{4} \mathrm{He}$. 


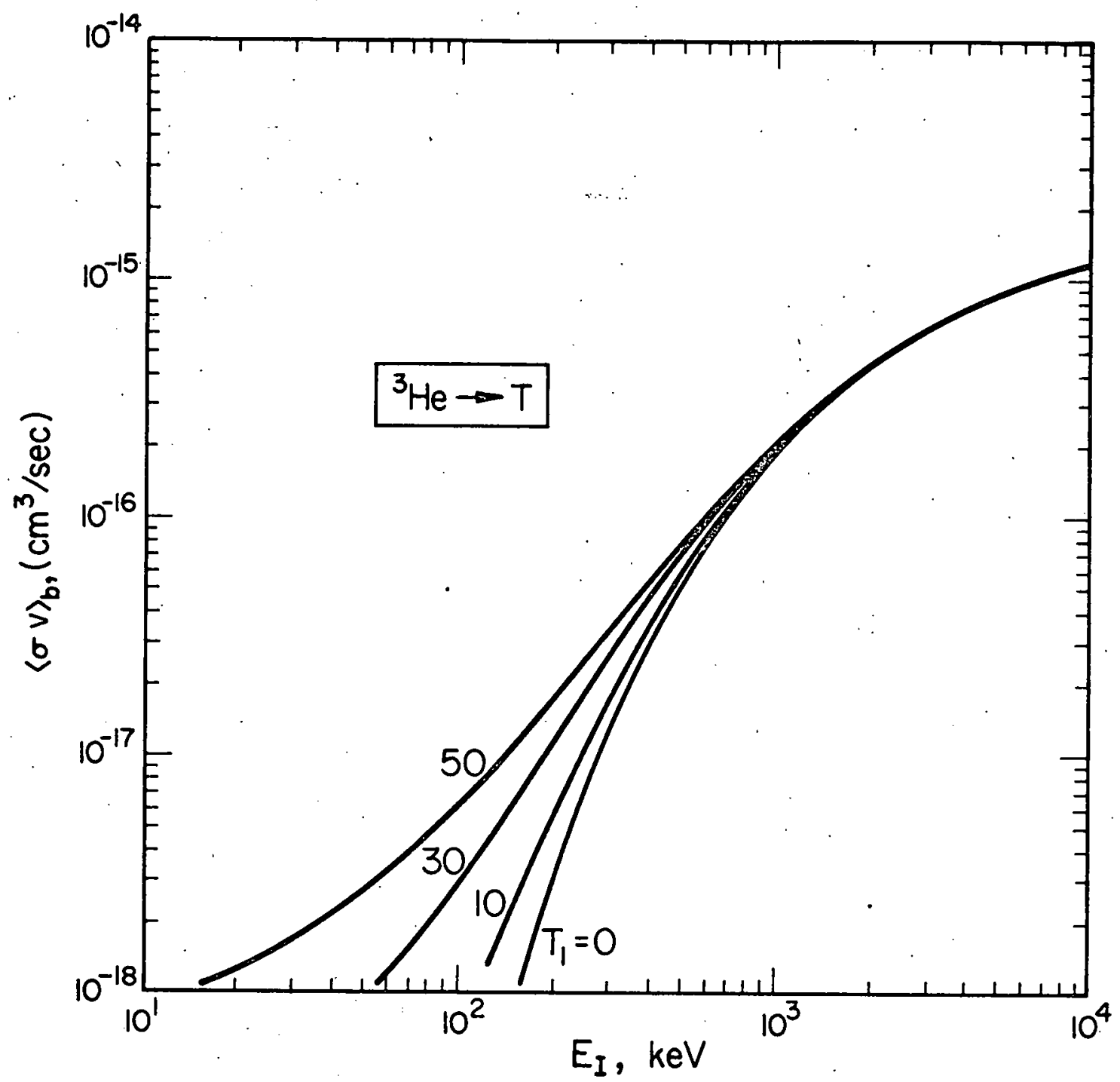

Fig. 1.5 Beam-Maxwellian reactivity for $\mathrm{T}\left({ }^{3} \mathrm{He}, \mathrm{x}\right) \mathrm{y}$ (where $x=p+n, d$, or $p$, and $y={ }^{4}$ He or ${ }^{5} \mathrm{He}$ ). 


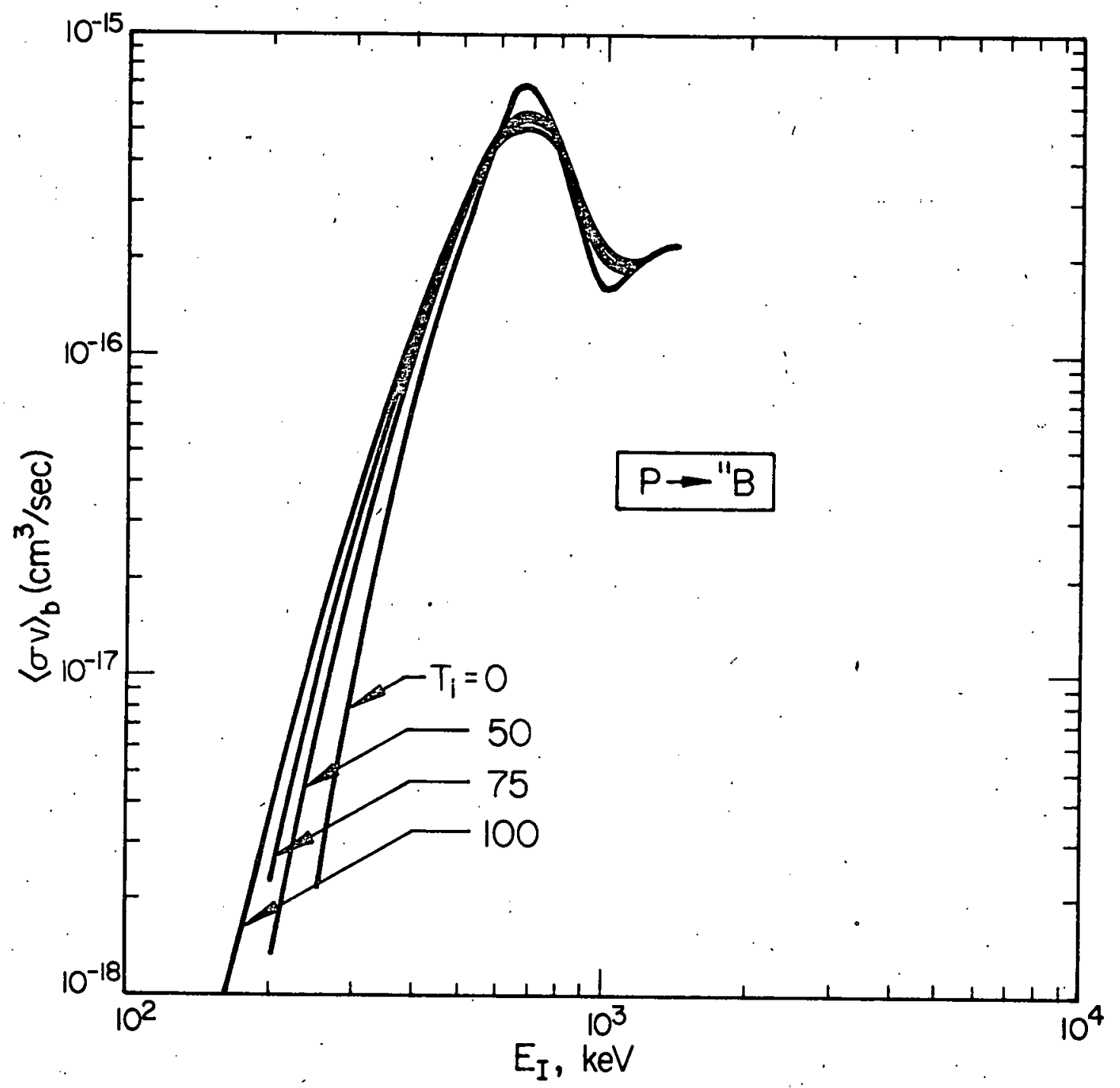

Fig. 1.6 Beam-Maxwellian reactivity for ${ }^{11} \mathrm{~B}\left(\mathrm{p}, 2^{4} \mathrm{He}\right)^{4} \mathrm{He}$. 
CHAPTER 2

ENERGY MULTIPLICATION FACTORS

\section{Introduction}

An important characterization of a two-component system is given by its energy multiplication factor defined as:

$$
F=\frac{E_{f}}{E_{I}}
$$

where $E_{f}=$ the energy released due to fusion reactions and $E_{I}=$ the initial energy of the injected ion.

\section{F-Value Calculation}

Since the energy of the injected ion continually decreases during thermalization, the fusion energy released per injected ion per unit volume can be written as:

$$
E_{f}=n_{i} Q_{F}\left[\int_{0}^{\tau} S D\langle\sigma v\rangle_{b} d t+\langle\sigma v\rangle \tau_{t h}\right] .
$$

Where: $n_{i}=$ number density of target ions which are responsible for fusion,

$\mathrm{Q}_{\mathrm{f}} \cdot \quad=$ energy released per reaction,

$\tau_{\mathrm{SD}}=$ the time it takes the injected particle to thermalize (slowing down time),

$\tau_{\text {th }}=$ the elapsed time between thermalization and when the particle leaves the system (thermal lifetime),

$\langle\sigma v\rangle_{b}=$ the fusion reaction rate parameter for beamMaxwellian interactions (see Chapter 1),

$\langle o v\rangle=$ the fusion reaction rate parameter for MaxwellianMaxwellian interactions. 
The first term in Eq. (2.2) gives the fusion energy contribution due to the injected ion slowing down. The second term gives the contribution to the fusion energy by thermalized ions. For early experiments in which the background ion temperature is $10 \mathrm{w}(\approx 10 \mathrm{keV})$ and the thermal lifetime is short $(\tilde{<} 1 \mathrm{sec})$, the latter term may be neglected.

By using Eq. (2.2) and the above assumption, -Eq. (2.1) can be written as:

$$
F=\frac{n_{i} Q_{f}}{E_{I}} \int_{0}^{\tau_{S D}}\langle\sigma v\rangle_{b} d t
$$

Under a change of variables, Eq. (2.3) is transformed into the following more useful form:

$$
F=\frac{n_{i} Q_{f}}{E_{I}} \int_{E_{t h}}^{E_{I}} \frac{\langle\sigma v\rangle_{b} d E}{|\langle d E / d t\rangle|}
$$

Where $E_{t h}=$ the thermalized energy of the injected ions (for numerical evaluation $E_{\text {th }}$ has arbitrarily been taken as twice the target ion temperature), and $\langle d E / d t\rangle=$ rate of change in energy of the injected particle.

In this work, $\langle\mathrm{dE} / \mathrm{dt}\rangle$ is based on the Fokker-Planck slowing-down model of Sivuhkin ${ }^{(13)}$, namely:

$$
\begin{aligned}
\left\langle\frac{d E}{d t}\right\rangle & =\frac{-4 \pi(Z e)^{2}}{v} \sum L^{*} n^{*}\left(Z^{*} e\right)^{2} \\
& x\left[\frac{\operatorname{erf}\left(b^{*} v\right)}{m^{*}}-\frac{2 b^{*} v\left(m^{*} m^{*}\right) e^{-b^{* 2} v^{2}}}{m^{*} \sqrt{\pi}}\right]
\end{aligned}
$$

where the sum is carried out over all species (ion and electrons), and: 


$$
\begin{aligned}
& b^{*}=\sqrt{m^{*} / 2 T^{*}} \\
& n^{*}=\text { number density of target species, } \\
& Z^{*}=\text { charge state of target species, } \\
& Z=\text { charge state of incident particles, } \\
& m=\text { mass of injected particle, } \\
& m^{*}=\text { mass of target species, } \\
& \mathrm{T}^{*}=\text { temperature of target species, } \\
& \mathrm{L}^{*} \equiv \text { the Coulomb logarithm. }
\end{aligned}
$$

The Coulomb logarithm is further split into a classical and a quantum-mechanical value depending on the magnitude of the relative speed. The forms for these two regions are:

I. The classical region:

$$
L_{c l}=\ln \sqrt{\left(\frac{\lambda_{d}}{\rho_{L}}\right)^{2}+1} \quad \text { Valid for } u \ll\left|z z^{*}\right| \alpha c
$$

where

$$
\begin{aligned}
& \lambda_{\mathrm{d}}=\text { Debye length, } \\
& \rho_{\perp}=\left|\frac{Z \mathrm{Z}^{*} \mathrm{e}^{2}}{\mu \mathrm{u}^{2}}\right|=90^{\circ} \text { scattering impact parameter, } \\
& \mathrm{u}^{2}=2 \mathrm{E} / \mathrm{m}+\frac{2 \mathrm{~T}^{*}}{\mathrm{~m}^{*}}=\text { average relative speed squared, } \\
& \mu \quad=\text { reduced mass }\left(\frac{\mathrm{mm}}{\mathrm{m}+\mathrm{m}^{*}}\right), \\
& \alpha \quad=\text { fine-structure constant }(1 / 137), \\
& \dot{c}=\text { speed of light (in a vacuum). }
\end{aligned}
$$

II. The quantum mechanical region:

$$
\begin{aligned}
L_{q m}=L_{c l}+\ln (2|z Z *| \alpha c / u)-1 / 2 & \\
& \text { Valid for } u \gg|z Z *| \alpha c
\end{aligned}
$$


In order to bridge the intermediate zone, the classical expression is extended until its value exceeds the quantum mechanical value, after which point the quantum mechanical expression is used.

With a finite ion temperature it is necessary to include the ion contribution to the Debye length. From Shkarofsky ${ }^{(14)}$ it is found that the total Debye length, $\lambda_{D}$, for a plasma containing $N$ species (ions and electrons) is given by:

$$
\frac{1}{\lambda_{d}{ }^{2}}=\sum_{j}^{N} \frac{1}{\lambda_{j}^{2}},
$$

where $\lambda_{j}$ is the normal Debye length for species $j, i . e$. $\lambda_{j}{ }^{2}=T_{j} / 4 \pi z_{j}{ }^{2} e^{2} n_{j}$. Note that due to the derivation of $\lambda_{j}$, the above result is only valid for $\left|z_{j} e \phi / T_{j}\right| \ll 1$, where $\phi$ is the electric potential. Hence, Eq. (2.8) is not valid for small $\mathrm{T}_{j}$. For the special case of $\mathrm{T}_{i}=0, \lambda_{\mathrm{d}}=\lambda_{\mathrm{e}}$ has been used here and in previous calculations by Dawson et al. (4) Because of this approximation a discontinuity arises between the zero and non-zero ion temperature cases. Still the gross features of the calculations are retained in the $\mathrm{T}_{i}=0$ case. (No attempt has been made to correct this problem since in practice a non-zero $T_{i}$ occurs and these calculations are all internally consistent.)

Figure 2.1 gives the rate of change in kinetic energy of deuterium as it slows down off of a pure tritium background at different temperatures. This figure illustrates the fact that as the plasma temperature is raised, $d E / d t$ for the incident particle decreases, resulting in an increased slowing down time. It is also seen that the electron drag term represents the dominate contribution at high kinetic energies while 


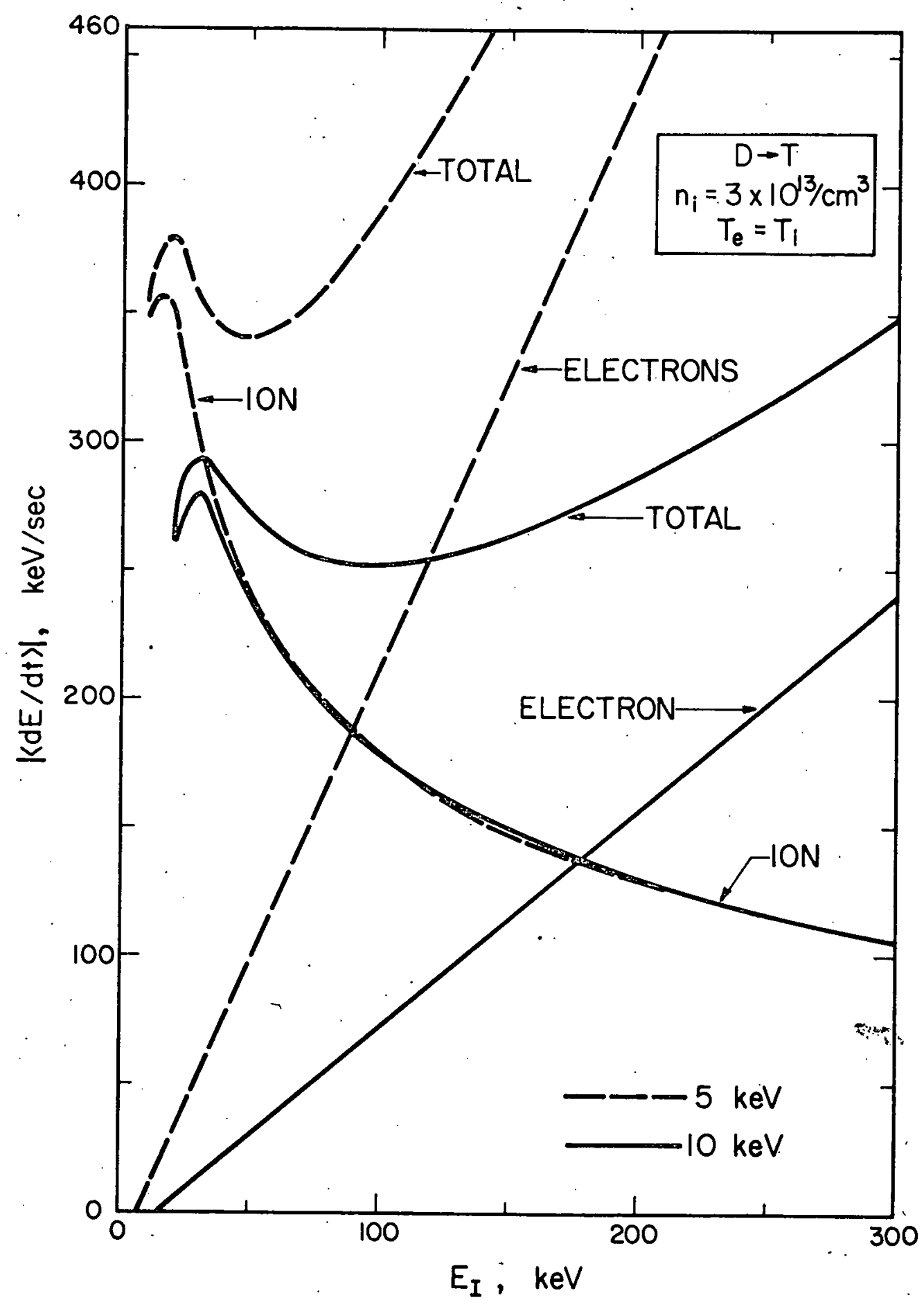

Fig. 2.1 Rate of change of kinetic energy for deuterium as it slows down in a pure tritium background. 
at low energies the ion drag dominates.

\section{G-Value Calculation}

Recently Furth and Jassby (15) suggested that instead of simply allowing the injected particles to slow-down by interactions with the background plasma, it might be advantageous to supply energy to the injected particles in order to maintain their energy, i.e. prevent slowing down (the beam is then said to be "clamped"). One possible way of achieving the desired energy input is through compression. $(15,16)$ The significance of the clamped mode as opposed to the earlier unclamped. conditions is that substantial increases in the energy multiplication factor are obtained. (15)

The energy multiplication factor for the clamped case is denoted as the G-value and is defined as:

$$
G=\frac{P_{f}\left(E_{I}\right) \tau_{h}}{E_{I}+(d E / d t){ }_{E}=E_{I} \tau_{h}},
$$

where $P_{f}=$ Power produced by fusion, $(d E / d t)_{E=E}=$ Power required to maintain injected particle at the energy $E_{I}$, and $\tau_{h}=$ Lifetime of the

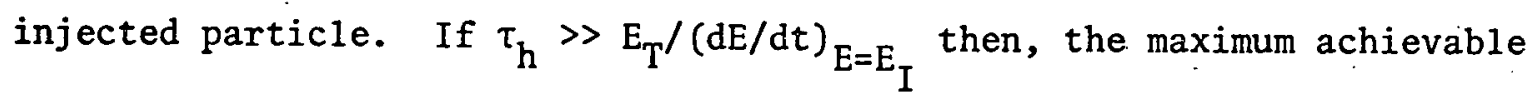
G-value $\left(G^{\prime}\right)$ is obtained and is given by:

$$
G^{\prime}=\frac{n_{i} Q_{f}^{\prime}\left\langle\sigma v{ }_{E_{I}}\right.}{(d E / d t)_{E=E_{I}}} .
$$

The G-values mentioned in the rest of this work will be defined by Eq. (2.10) and hence the prime will be deleted. 
Results

Figures 2.2 and 2.3 show the general trends for $F^{*}$ and G-values as a function of injection energy for deuterium injected into pure tritium and ${ }^{3}$ He targets respectively. Here it is assumed that $T_{e} \sim T_{i}$ which is not too different from the situation expected in the TCT (see Chapter 3). The two temperature levels (5 and $15 \mathrm{keV}$ ) are representative of early experimental devices** and later reactors, respectively. The target plasma density in this and subsequent figures is taken as $210^{14}$ ions $/ \mathrm{cm}^{3}$. However, energy multiplication is quite insensitive to the target density, e.g. calculations show that F-values increase only of the order of $10 \%$ as the density of the tritium target is increased from $10^{13}$ to $10^{17}$ ions $/ \mathrm{cm}^{3}$. This is because, to first order, the fusion rate and the energy loss rate for the injected ions are both linear functions of density. Since $F$ and $G$ involve ratios of these quantities, the density dependence roughly cancels out.

To gain some insight into the magnitudes of $F$ and G-values of interest, we note that if all of the fusion output were processed through thermal and direct converters (efficiencies $\eta_{\text {th }}$ and $\eta_{\mathrm{DC}}$, respectively) and recirculated to the injector, the minimum F-value for a self-sustain-

The $\mathrm{D} \rightarrow \mathrm{T}$ F-values in this chapter use $\mathrm{Q}_{f}=22.4 \mathrm{MeV}$ which includes $4.8 \mathrm{MeV}$ for blanket reactions.

For a "break-even" energy ATC-type device, design goals are $\mathrm{T}$ i 3.3
$\mathrm{keV}, \mathrm{n \tau} \sim 5 \times 10^{12} \mathrm{~cm}^{-3} \mathrm{sec}$, and 2.2 compression ratio(16). 


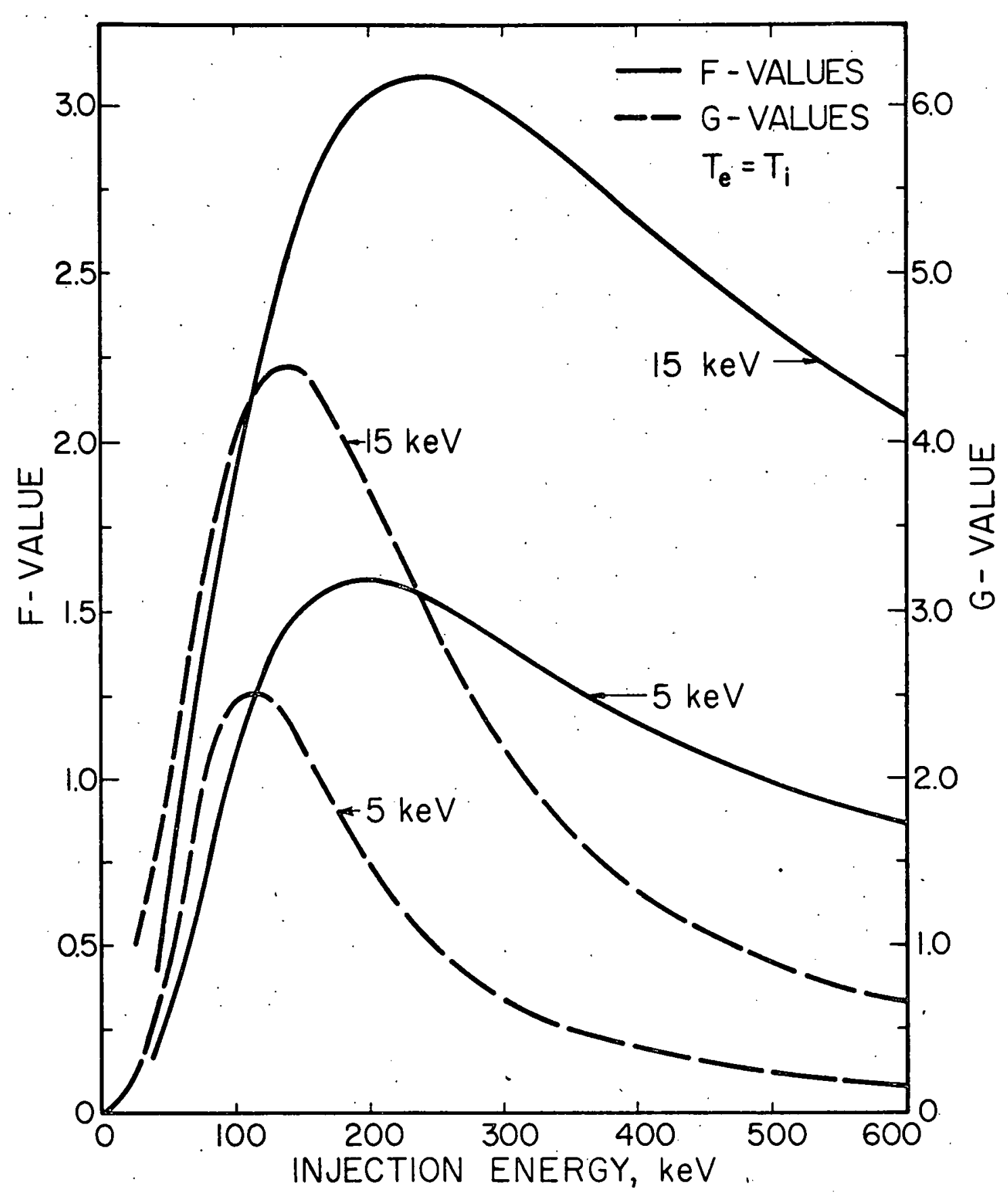

Fig. 2.2 F- and G-values for injection of deuterium into a pure tritium target plasma with $1 \times 10^{14}$ ions $/ \mathrm{cm}^{3}$. 


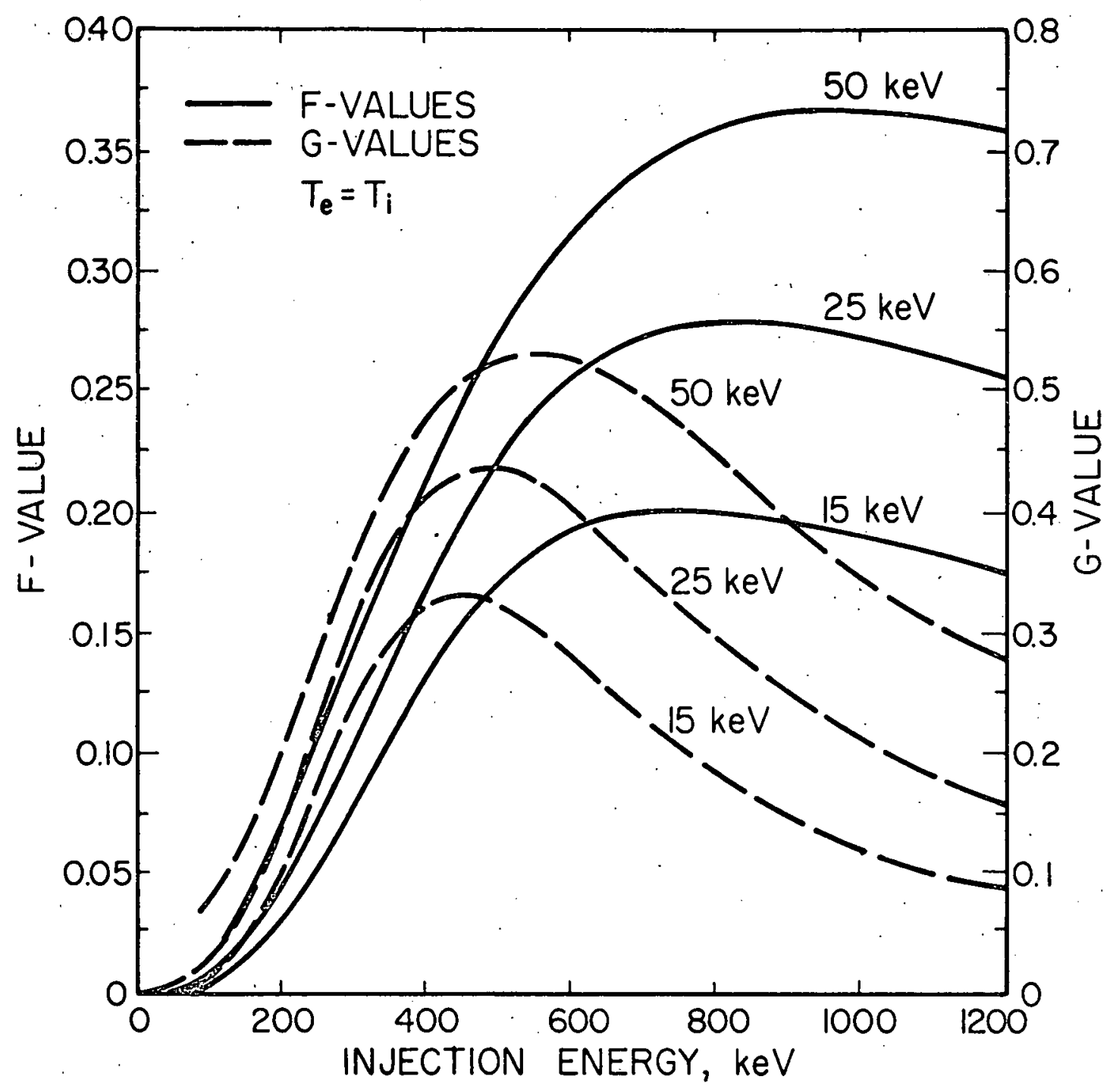

Fig. 2.3 F- and G-values for injection of deuterium into a pure $\mathrm{He}^{3}$ target plasma with $1 \times 10^{14}$ ions $/ \mathrm{cm}^{3}$. 
ing systèm is* (see Fig. 2:4):

$$
F_{\min }=\frac{\eta_{I}^{-1}-\eta_{D C}}{\eta_{D C}-f_{n}\left(\eta_{D C}-\eta_{t h}\right)}
$$

Here $f_{n}$ is the fraction of the fusion energy going into neutrons ( 0.8$)$ for $\mathrm{D}-\mathrm{T}$ and $\mathrm{iO}_{\mathrm{O}}$ for $\mathrm{D}-\mathrm{He}^{3}$ ), and we have neglected radiation losses and assumed that all fusion product energy is contained within the plasma. Note that the well-known Lawson criterion corresponds to D-T fuel with $\eta_{I}$, the injection efficiency, equal to $100 \%$, and $\eta_{D C}=\eta_{\text {th }}=33 \%$, gives $F_{\text {Lawson }}=2.0$. Other $\mathrm{F}_{\text {min }}$-values for typical conversion conditions are summarized in Table 2.1 .

It would be very difficult to build a near term experiment with efficiency levels for $\eta_{I}, \eta_{D C}$, and $\eta_{\text {th }}$ over $60 \%, 33 \%$, and $33 \%$ respectively. Fortunately, this is not necessarily the goal. Rather, the objective is to obtain plasma conditions i.e. F-values such that break even would indeed be possible with anticipated future injector and conversion efficiencies. In fact the early experiments may not even use an energy converter. In that case the input power would be purchased and the fusion energy dissipated in a heat dump. In this light, Table 2.1 suggests a F-value goal of 1.5-2.0 for D-T, or alternately, 0.6 to 1.0

*In Ref. 16 the breakeven $F$ (or $G$ ) value is given as $G=\left(n^{-1}-1\right)$ where $\eta$ is the net energy recycling efficiency. To compare this to Eq. (11) we note that if $n_{D C}=\eta_{t h}$ then

$$
F_{\text {min }}=\frac{1}{\eta_{I} \eta_{t h}}-1
$$

or $\eta \equiv \eta_{I} \eta_{\text {th }}$. Thus, the case $\eta_{I}=0.6$ and $\eta_{\text {th }}=0.33$ in Table 3.2 corresponds to $n=0.2$ while $\eta_{I}=0.9$ and $\eta_{\text {th }}=0.4$ gives $n=0.036$. The latter is slightly lower than the $\eta=0.4$ assured in Ref. 16 which has been used to set the goal of a F or G minimum of 1.5 . 


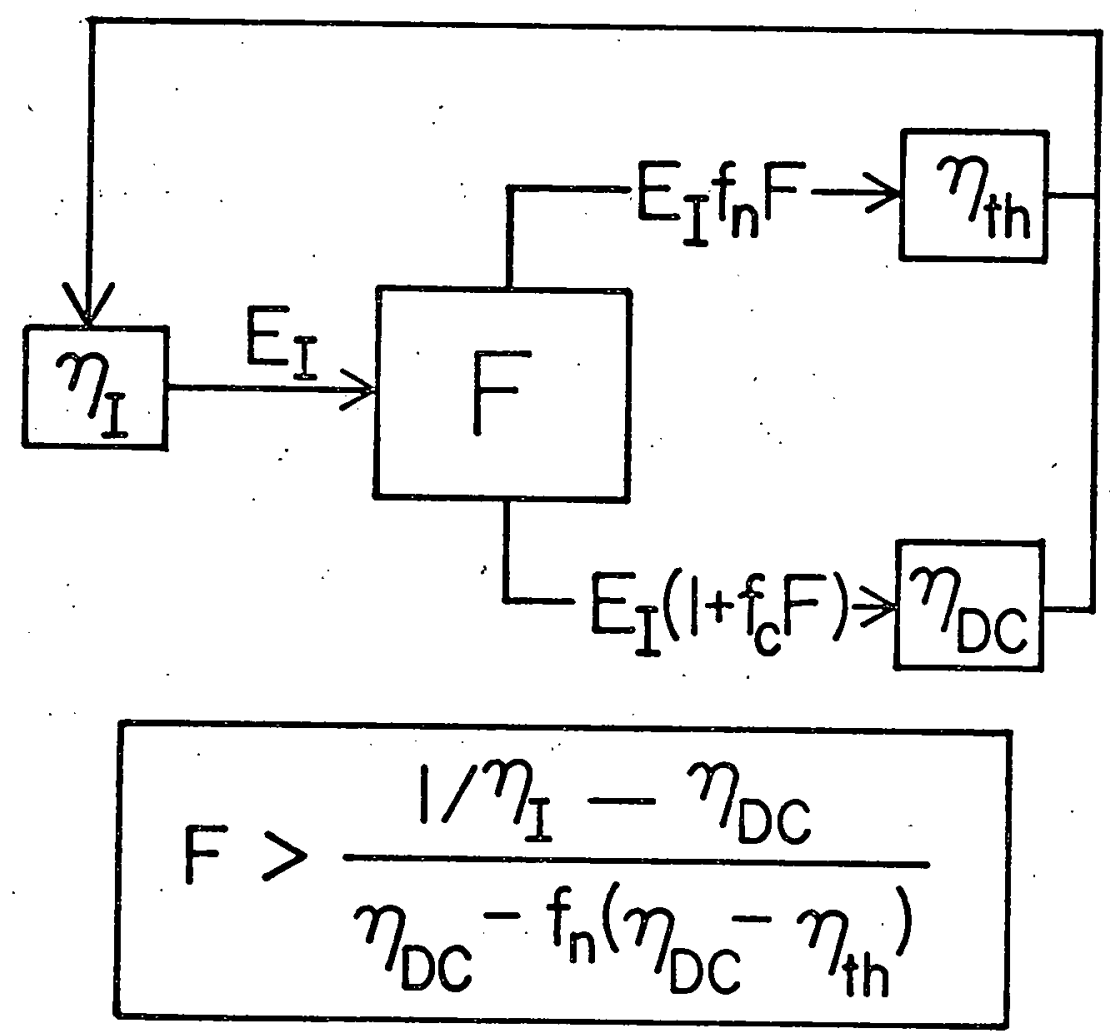

Fig. 2.4 Idealized energy flow diagram for evaluation of $\mathrm{F}_{\min }^{*}$ 
Table 2.1 Minimum F-values For A Self-Sustaining System (Complete recirculation of output power)

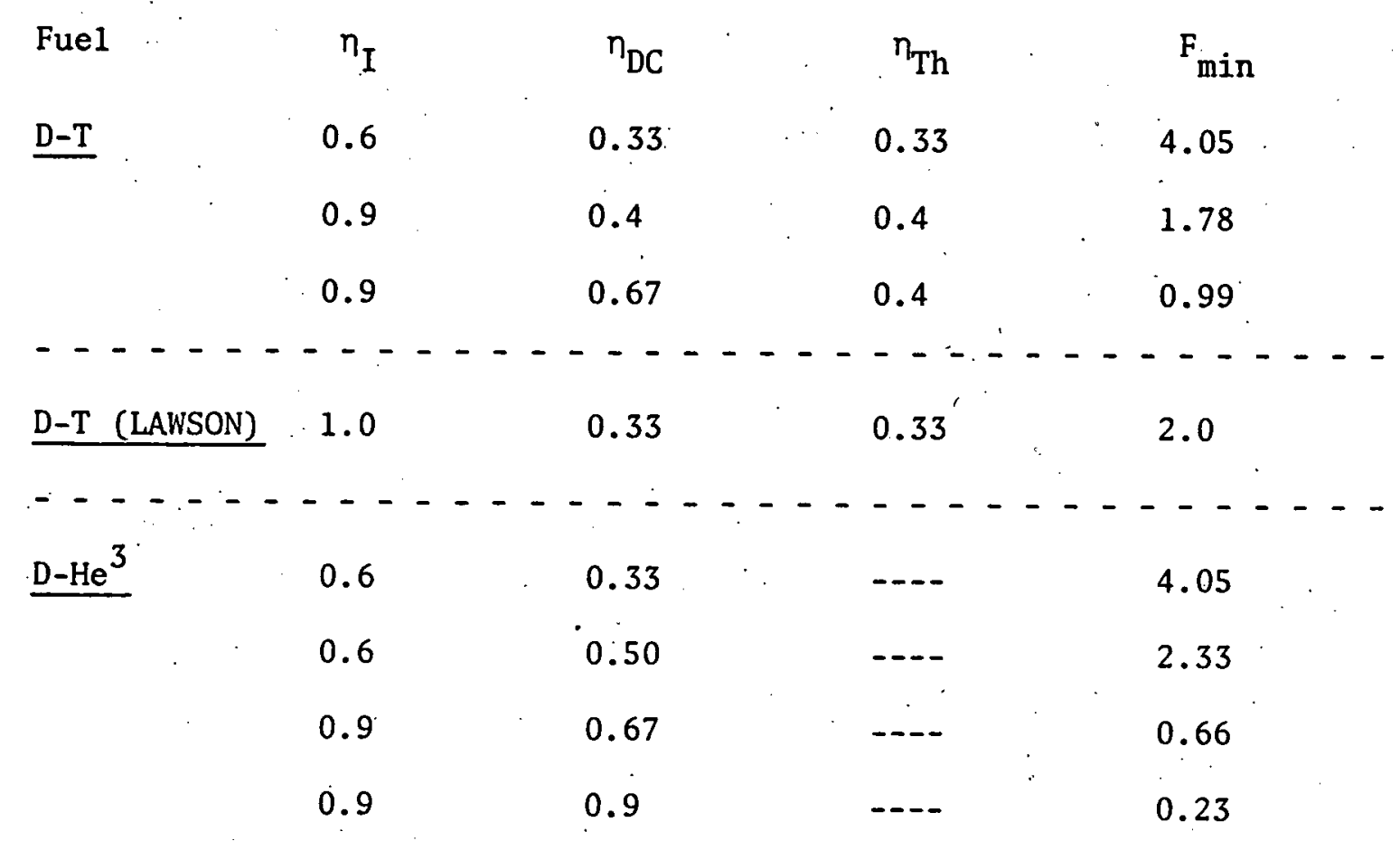


for $\mathrm{D} \rightarrow \mathrm{He}^{3}$. From Figs. 2.2 and 2.3 (also see Figs: 2.10 and 2.11 ), this translates into $\mathrm{T}_{\mathrm{e}}$ values of 5 to $10 \mathrm{keV}$ for $\mathrm{D} \rightarrow \mathrm{T}$ but does not appear to be practical for $\mathrm{D} \rightarrow \mathrm{He}^{3}$. (For ultra-high efficiencies $\mathrm{T}_{\mathrm{e}}>25 \mathrm{keV}$ could work for $\mathrm{D} \rightarrow \mathrm{He}^{3}$, but this would require considerable advances in technology).

The use of clamping would relax these requirements even further. If a compression scheme such as proposed in Ref. 16 is employed, it is found that for tangential injection into a plasma of $10 \mathrm{w} \mathrm{T}_{e}$ and $\mathrm{Z}=1$, *

$$
G=\frac{G_{\max }\left(T_{e}\right)}{1+\left(2 C^{2} \ln C\right)^{-1}}
$$

Here $\mathrm{C}$ is the compression ratio during the burn, and this result corrects the maximum G-value (the value read from figures in this report) for the additional energy input supplied to the plasma by compression. Thus, $G$ from Eq. (2.12) is equivalent to the F-value discussed earlier and Eq. (2.11) and Table 2.1 can now be interpreted in terms of the minimum requirement for $\mathrm{G}$ from $\mathrm{Eq} .(2.12)$.

A plot of $G / G_{\max }$ vs. C based on Eq. (2.12) is shown in Fig. 2.5 . If, for example, a compression ratio of 2 were achieved during the burn, $G / G_{\max }$ is roughly 0.8 . Using this and the $G$-value plots we see that this reduces the $T_{e}$ values required for $D-T$ experiments from the $5-10 \mathrm{keV}$ region found earlier to $3-5 \mathrm{keV} . * *$ (Also, as shown later in Fig. (2.10)

More realistic expressions are given in Ref. 16 for isotropic injection, high-T $\mathrm{T}$ or a $Z>1$ plasma as well as for other compression schemes. However for simplicity, Eq. (3.12) is used here to provide order of magnitude estimates. The latter gives $\mathrm{G} / \mathrm{G}_{\max }$ ratios $\sim 10 \%$ or so higher
than the more general expression.

**A more complete discussion of a breakeven experiment in Ref. 16 gives: $T_{e}=3.0 \mathrm{keV}, E_{I}=110 \mathrm{keV}, C=2.3$, and $n_{e o} \tau_{c}=4 \times 10^{12} \mathrm{~cm}^{-3}$ sec. 


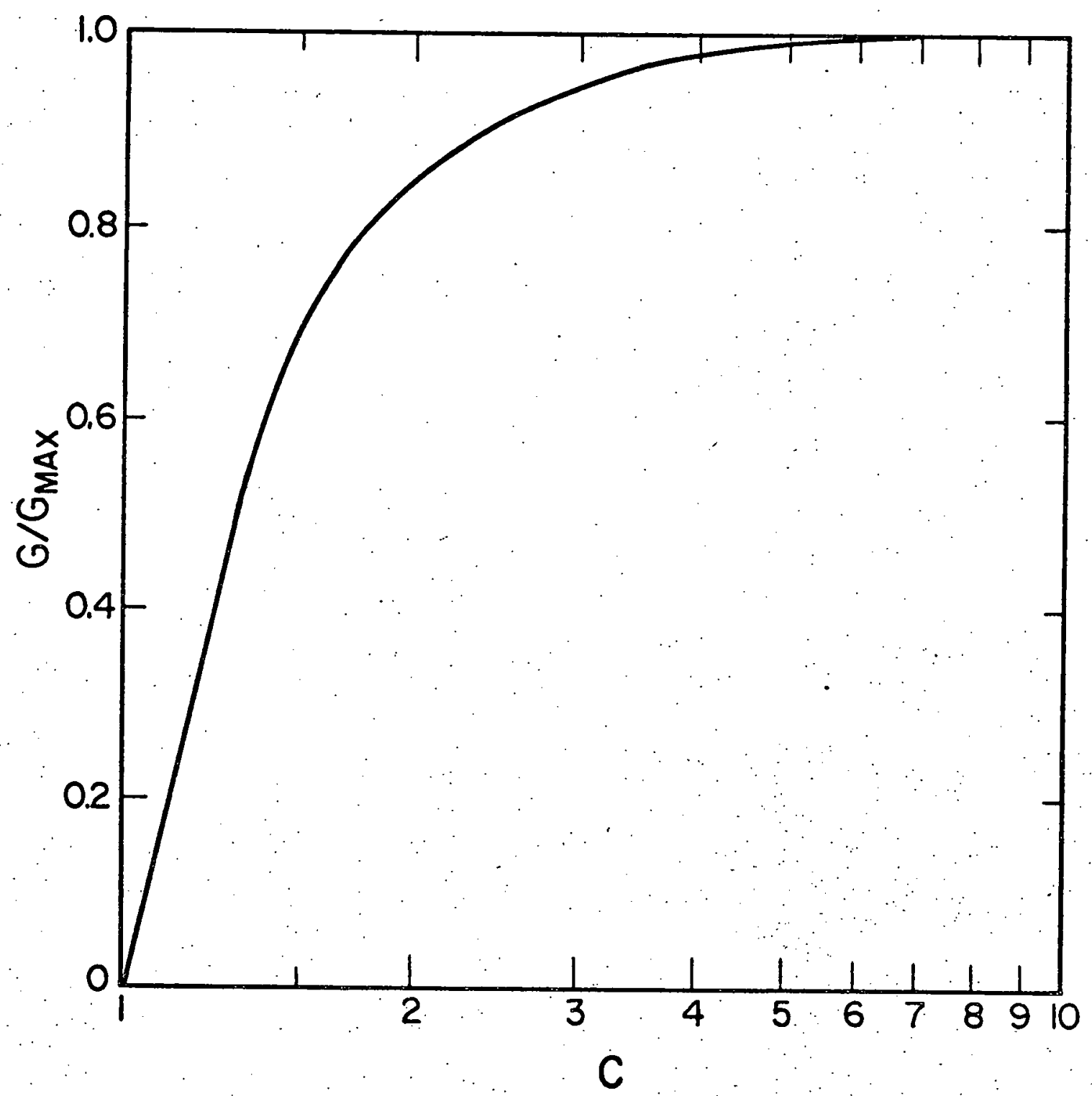

Fig. 2.5 Influence of compression ratio on the ratio $\mathrm{G} / \mathrm{G}_{\max }$ for idealized tangential injection. 
the injection energies for maximum multiplication are reduced from i $180 \mathrm{keV}$ to $\sim 110 \mathrm{keV}$ ). Also $\mathrm{D} \rightarrow \mathrm{He}^{3}$ becomes a more realistic possibility although this needs more study. At any rate, the motivation for clamping, even in early experiments is clear from these examples.

In some cases, particularly at higher temperatures where an additional contribution from the thermal fusions background can occlir, it may be of interest to inject deuterium into a D-T mixture. F- and Gvalues for this case are shown in Fig. 2.6.* Since the D-D contribution is small at the lower energies, the maximum multiplication scales in a nearly. linear fashion with the percent tritium in the target as seen from Fig. 2.7.

The question of the effect of non-zero target-ion temperature will now be discussed. Figure 2.8 shows F- and G-values for injection of deuterium into pure tritium for $T_{e}=15 \mathrm{keV}$ and three ion temperatures $(5,10$ and $15 \mathrm{keV})$. The shapes of the curves are all similar, but the multiplication is reduced by the increased ion temperature for large injection energies. While the slowing-down time increases slightly with increasing $\mathrm{T}_{i}$ ( a positive contribution to the F- and G-values) the dominant effect is due to the reduced fusion reaction rate (see. Fig. 1.1)

As seen from Fig. 2.9, the ion-temperature effect can cause as much as a $10 \%$ reduction in $F$ and $G$ over this range of temperatures. Another way of viewing this is as follows: If, in a $\mathrm{T}_{\mathrm{e}}=10 \mathrm{keV}$ plasma, the ion temperature turns out to be $10 \mathrm{keV}$ instead of $3 \mathrm{keV}$,

\footnotetext{
*These results are for the beam-target fusion only. Additional fusion occurring in the target is neglected, and in fact, for simplicity, $\mathrm{T}_{\mathbf{i}}=0$ is used:
} 


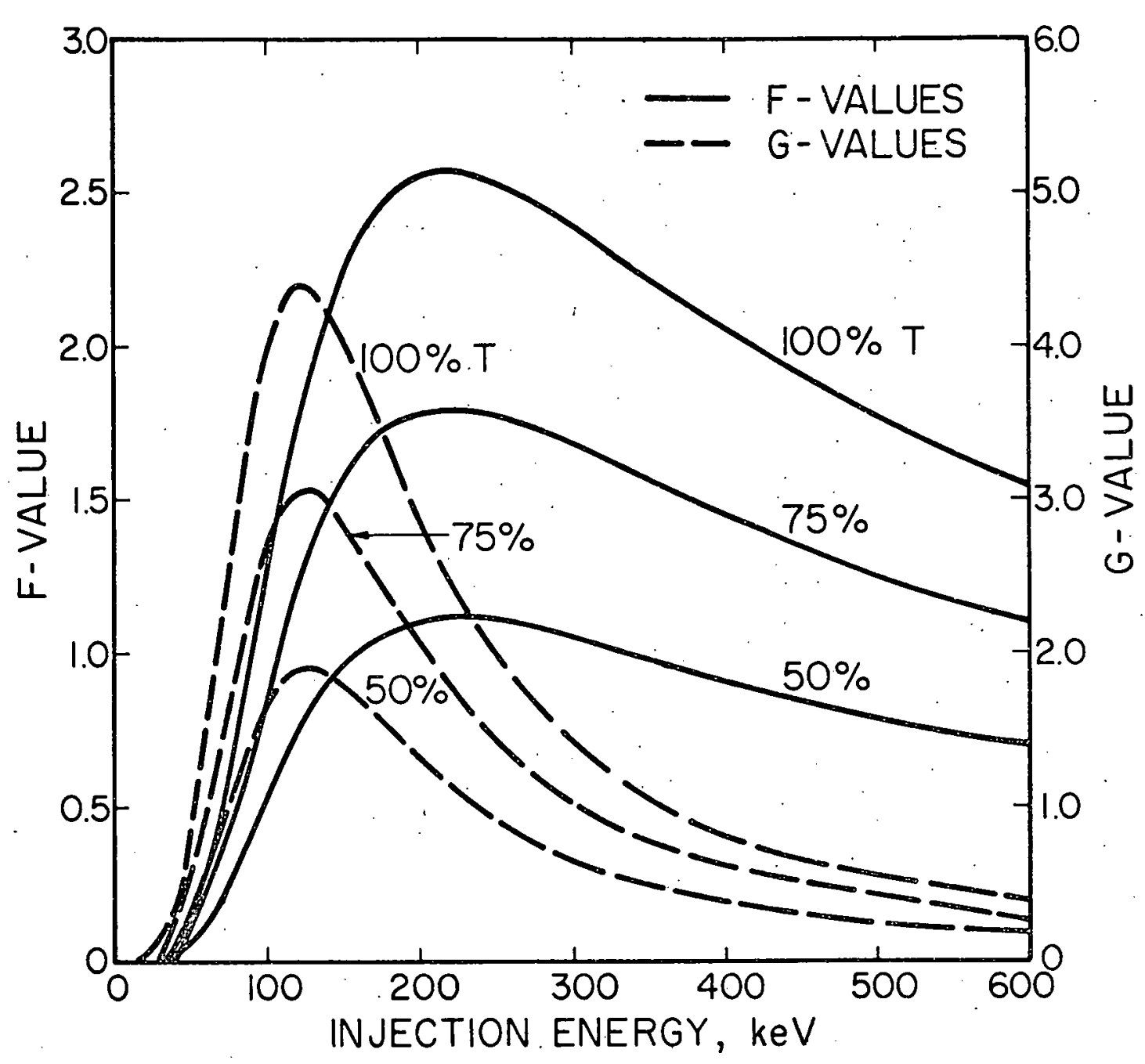

Fig. 2.6 F- and G-values for injection of deuterium into various mixtures of deuterium and tritium target plasma with $\mathrm{T}_{3} \mathrm{e}=10 \mathrm{keV}$, $\mathrm{T}_{i}=0$ and $1 \times 10^{14}$ ions $/ \mathrm{cm}^{3}$. 


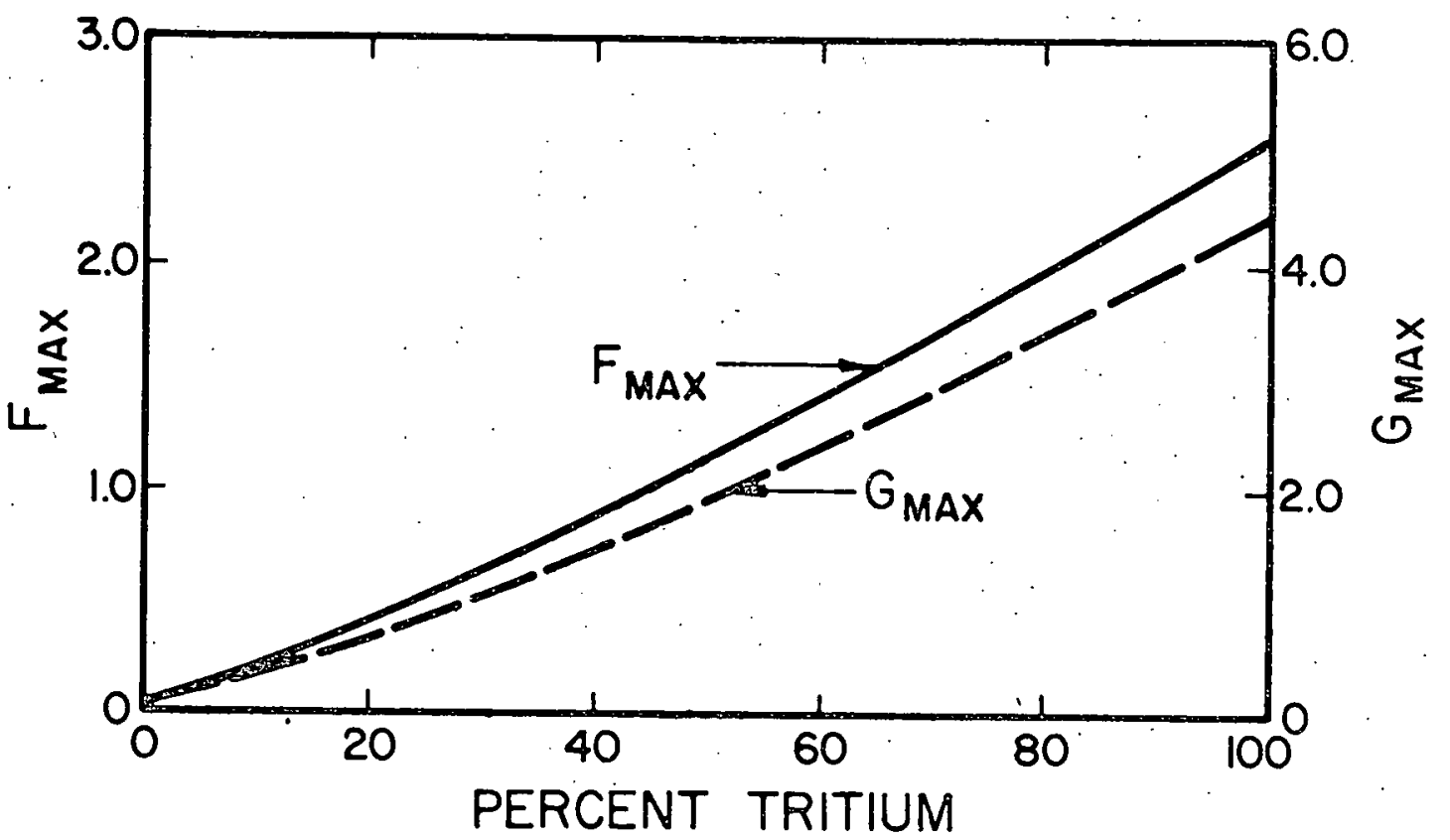

Fig. 2.7 Maximum F- and G-values for injection of deuterium into various mixtures of deuterium and tritium target plasma with $\mathrm{T}_{\mathrm{e}}=10 \mathrm{keV}$, $\mathrm{T}_{i}=0$ and $1 \times 10^{14}$ ions $/ \mathrm{cm}^{3}$. 


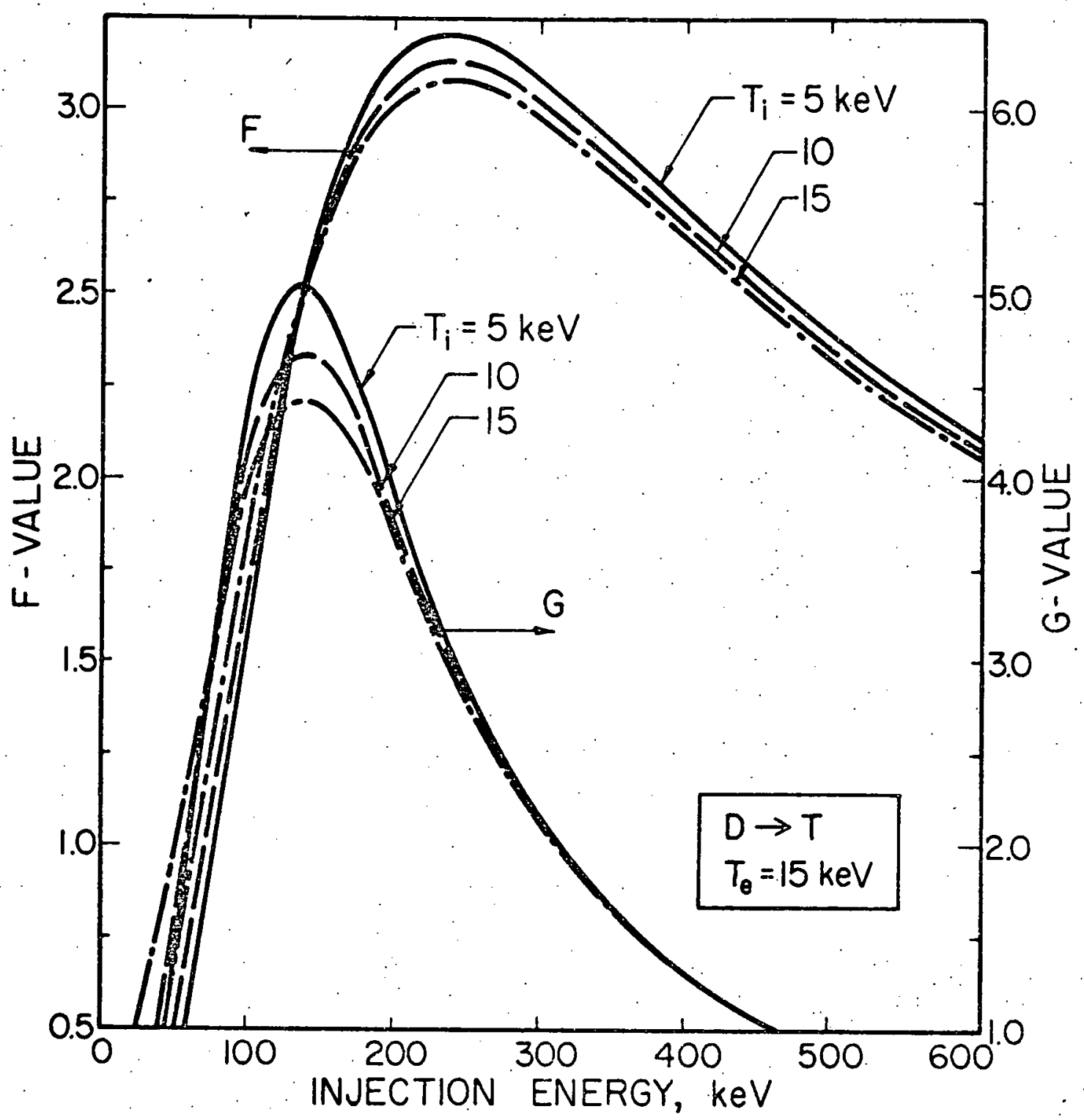

Fig. 2.8 F- and G-values for injection of deuterium into a pure tritium target plasma with various $T_{i}$ but $\mathrm{T}_{\mathrm{e}}=15 \mathrm{keV}$ and $1 \times 10^{14}$ ions $/ \mathrm{cm}^{3}$. 


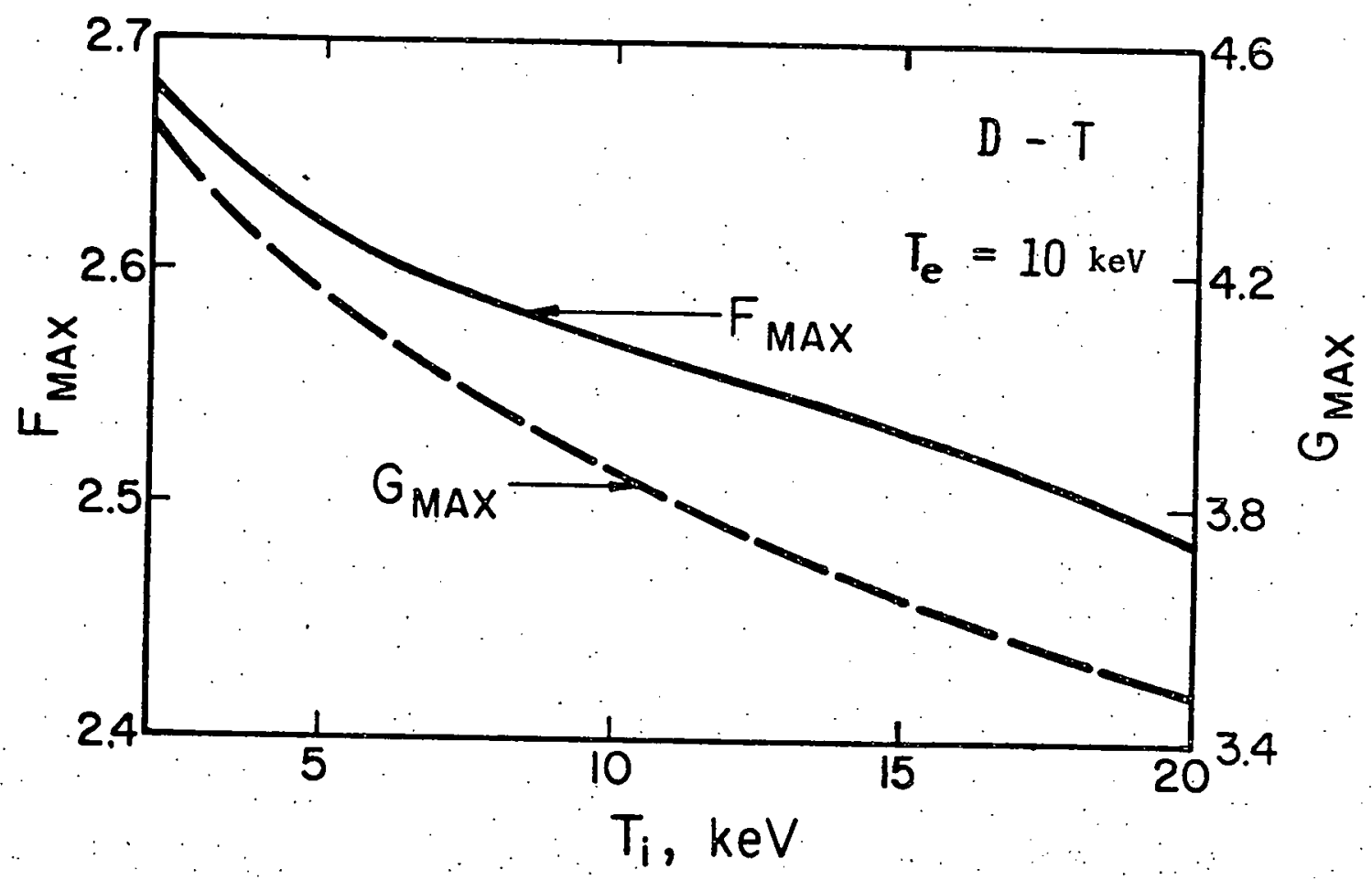

Fig. 2.9 Maximum F- and G-values for injection of deuterium into a pure tritium target with $\mathrm{T}_{\mathrm{e}}=10 \mathrm{keV}$ and $1 \times 10^{14}$ ions $/ \mathrm{cm}^{3}$. 
the maximum $F$ and $G$ values would decrease from 2.68 to 2.48 and 4.5 to 3.7, respectively. As shown next in Fig. 2.10, this could be roughly compensated for by increasing $\mathrm{T}_{\mathrm{e}}$ from 10 to $14 \mathrm{keV}$.

The remaining two figures $\left(2.10\right.$ and 2.11 ) show $G_{\max }$ and $F_{\max }$ vs. $T_{e}$ and the corresponding injection energies for $D \rightarrow T$ and $D \rightarrow H e^{3}$, respectively. Curves for the extremes of $T_{i}=0$ and $T_{i}=T_{e}$ are shown, and it is observed that for $\mathrm{D} \rightarrow \mathrm{T}$. the ion temperature effect is negligible for early experiments where $\mathrm{T}_{\mathrm{e}}$ is expected to be below $5 \mathrm{keV}$. However, in the range of eventual interest above $10 \mathrm{keV}$, it must be considered as illustrated by the previous example. For $\mathrm{D} \rightarrow \mathrm{He}^{3}$ the most important effect is in $G_{\max }$ above $220 \mathrm{keV}$, but this too is the region of ultimate interest for this fuel.

Summary

The $\mathrm{T}_{\mathbf{i}}=0$ values presented in this work agree reasonably, well with the calculations of Furth and Jassby (15). The largest discrepancies. found are for the high temperature F-values which differ at the most by $13 \%$

In interpreting these results, especially where the higher ion temperatures are involved, it must be remembered that all energy multiplication factors presented here only include the contribution due to beam-target fusion, i.e. any "steady-state" fusion after the injected particles have thermalized has been neglected.

In conclusion, these results show that finite ion temperature will not adversely affect early experiments. However; as the reactor regime is approached, this question as well as the proper incorporation of fusion in the target plasma deserve more study. 

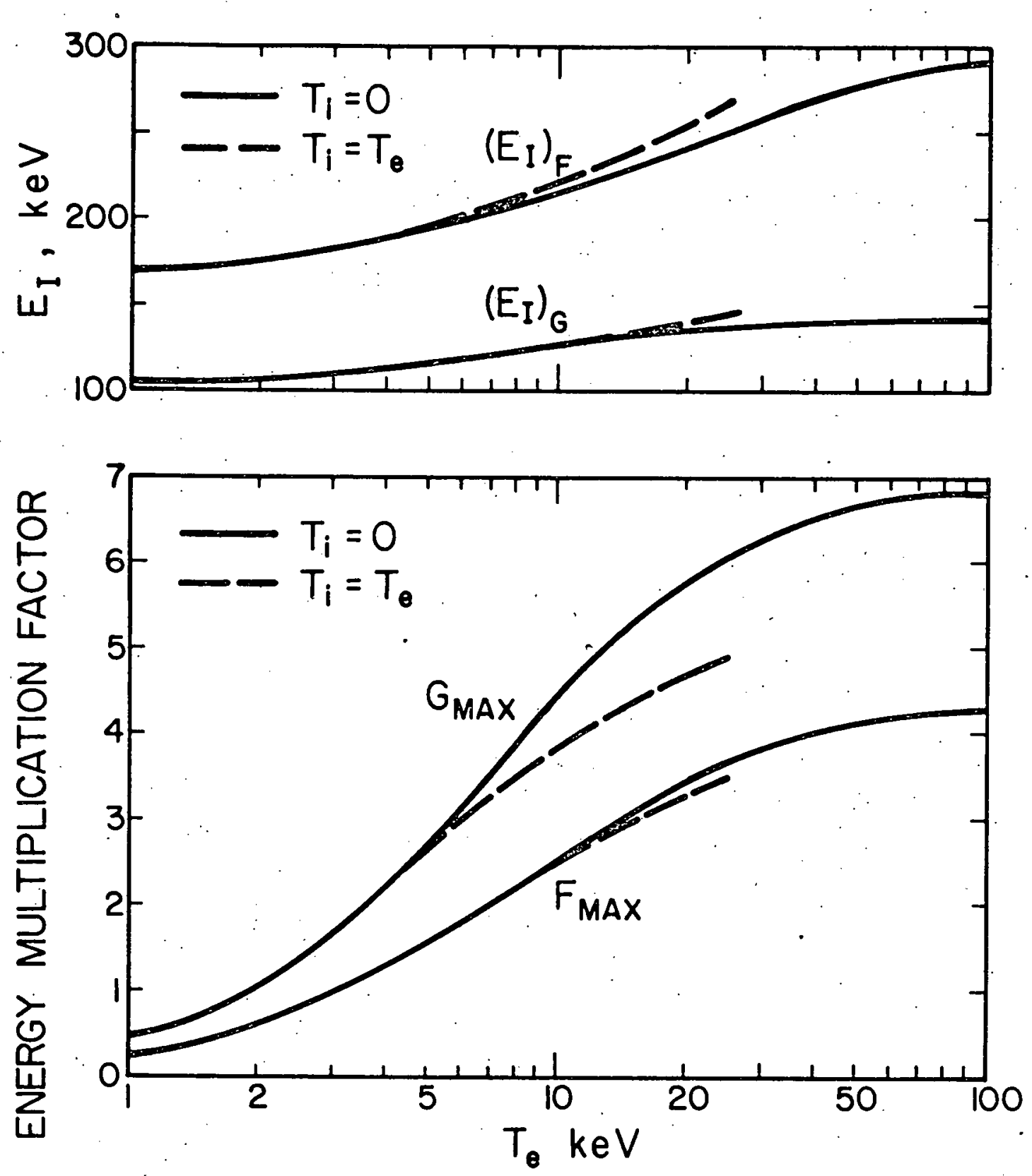

Fig. 2.10 a: Optimal injection energies for a clamped and unclamped deuterium beam incident upon a pure tritium target plasma with $1 \times 10^{14}$ ions $/ \mathrm{cm}^{3}$.

b. Optimal energy multiplication factor for clamped and unclamped beams. 

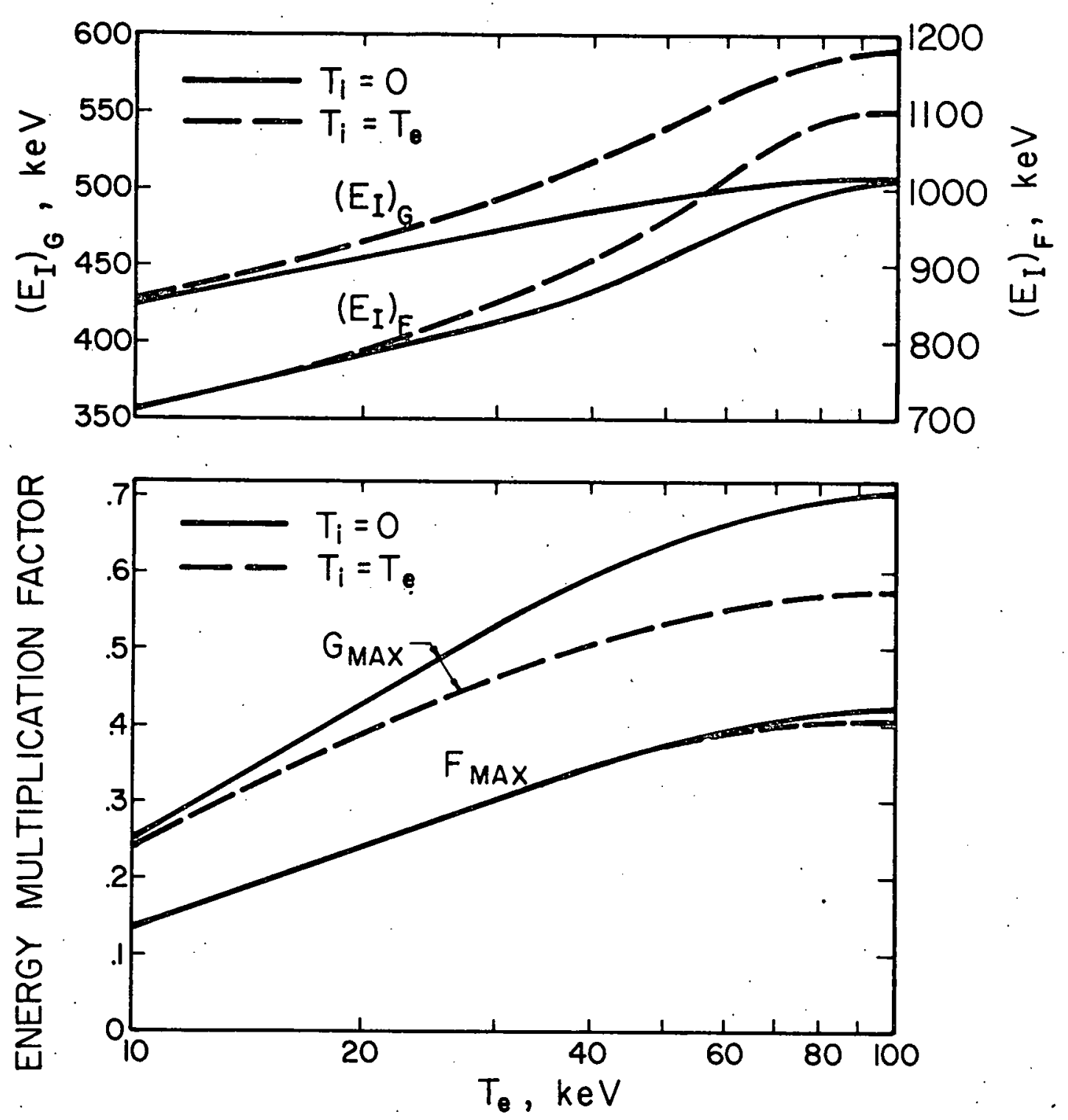

Fig. 2.11 a. Optimal injection energies for clamped and unclamped deuterium beam incident upon a pure $\mathrm{He}^{3}$ target plasma with $1 \times 10^{14}$ ions $/ \mathrm{cm}^{3}$.

b. Optimal energy multiplication factor for clamped and unclamped beams. 
The energy multiplication values for $\mathrm{D} \rightarrow \mathrm{T}$ appear to be quite favorable for early experiments. There is sufficient margin of safety so that even if additional detailed effects are found that decrease $F$ and $G$ below values shown here, the probability for success seems good. While $\mathrm{D} \rightarrow \mathrm{He}^{3}$ values are also included here, the results appear to be marginal. Still, the reduced neutron and radioactivity hazards might provide important time and money savings in early experiments so the possibility should not yet be discarded. In fact, for a burner experiment, other considerations may be more important than breakeven. Since a major physics question involved is the effect of fusion product heating on such factors as stability; we might ask what conditions are required to test the stability of the two-component system. This might be studied if the ratio of pressures due to superthermal ions vs. background plasma pressure for $D \rightarrow T$ could be roughly duplicated in the $D \rightarrow H e^{3}$ experiment. Preliminary results show that this might be feasible if relatively low background ${ }^{3} \mathrm{He}$ densities are employed, and in this light, the preliminary D- ${ }^{3} \mathrm{He}$ experiments become more interesting. 
CHAPTER 3

POWER BALANCE

\section{Introduction}

In the previous chapters, parameters were varied one at a time to demonstrate trends. In practice, however, interrelationships between the various parameters must be considered. The present chapter considers the energy multiplication in a plasma where the background ion and electron temperatures $\left(\mathrm{T}_{i}\right.$ and $\mathrm{T}_{\mathrm{e}}$ respectively) are found from selfconsistent energy balances involving the injected energy $\left(E_{I}\right)$, injection rate $\left(j_{I}\right)$, and the particle and energy confinement times.

The Model

The required'relationship between $E_{I}, j_{I}, T_{i}$ and $T_{e}$ can be derived from the simplified ion-electron power balance depicted in Fig. 3.1. In this figure a steady-state energy condition is achieved by a balance between the incoming and outgoing power densities associated with both ions and electrons.* A steady-state particle balance is maintained by demanding that the rate of input of a given species exactly matches their loss rate.

In this model the incoming power density is the sum of the powers transferred to the plasma ions and electrons from the injected beam $\left(P_{I, i}\right.$ and $\left.P_{I, e}\right)$ and from the charged fusion products $\left(P_{f, i}\right.$ and $\left.P_{f, e}\right)$. Note that ohmic heating has been neglected since this makes up a small fraction of the incoming power density (see Ref. 2). The outgoing power

"The TCT will operate in a pulsed mode; however, the present balances should be valid over a major portion of the pulse where changes with time are sufficiently slow such that a quasi-steady state plasma is attained. 


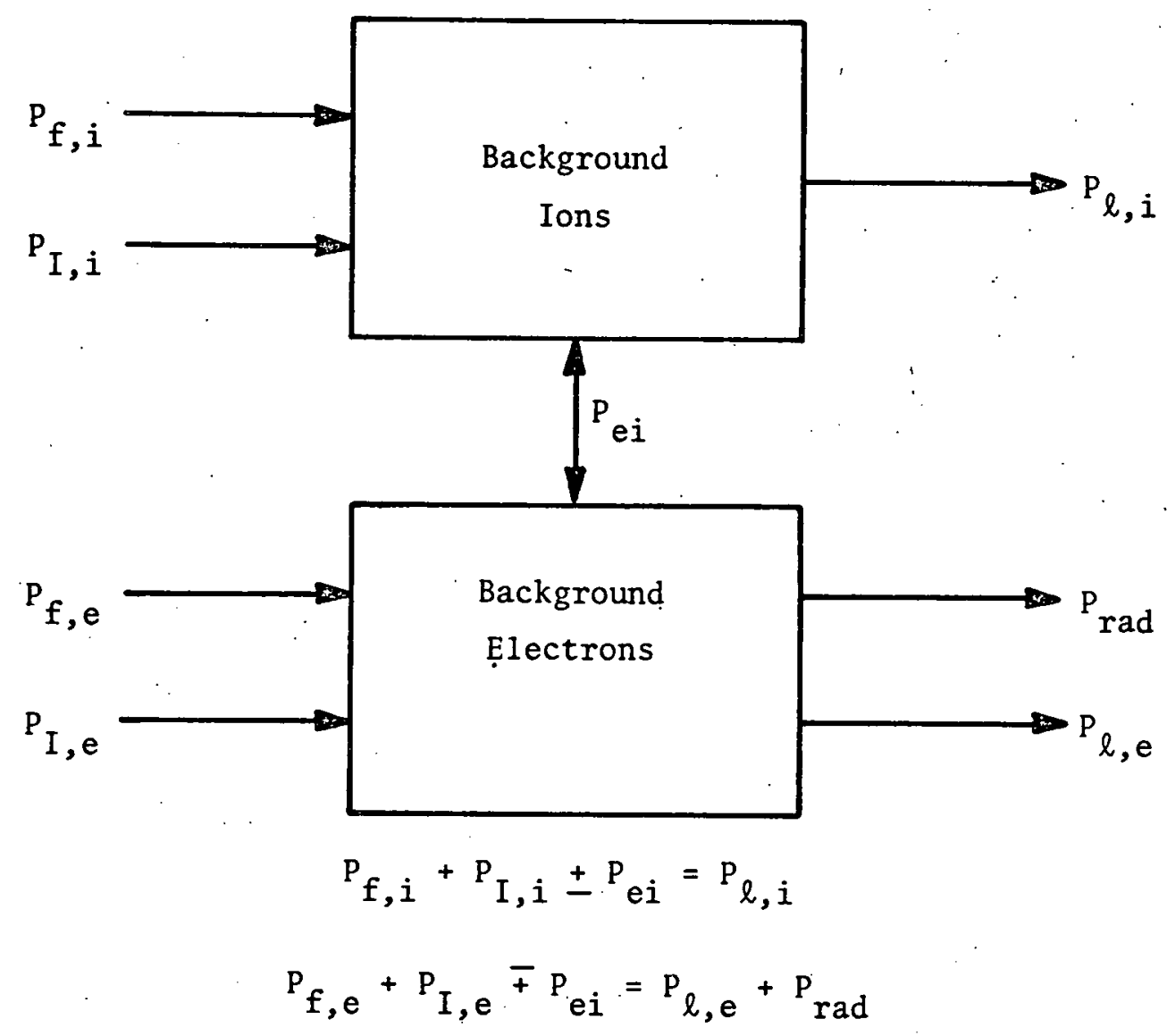

Fig. 3.1 The power balance employed in TCT calculations. The following nomenclature is employed:
$P_{f, i(e)}-\begin{aligned} & \text { Power } / \mathrm{cm}^{3} \text { transferred from } \\ & \text { fusion products to the }\end{aligned} \mathrm{P}_{\text {rad }}-$ The radiation power $/ \mathrm{cm}^{3}$. background ions (electrons).
$\mathrm{P}_{\mathrm{I}, \mathrm{i}(\mathrm{e})}$ - Power $/ \mathrm{cm}^{3}$ transferred from the injected beam to back- ground ions (electrons).
$P_{\text {ei }}$ - The exchange power $/ \mathrm{cm}^{3}$ between electrons and ions.

$$
\begin{aligned}
& \mathrm{P}_{\ell, i(e)}- \text { The leakage power } / \mathrm{cm}^{3} \\
& \text { associated with the } \\
& \text { background ions } \\
& \text { (electrons). }
\end{aligned}
$$


density is the sum of the radiation power density $\left(\mathrm{P}_{\text {rad }}\right.$ ) (only for electrons), and the leakage power density $\left(P_{\ell, i}, P_{\ell, e}\right)$. The latter accounts for the power which is physically transported out of the plasma with the escaping plasma plus that lost by thermal conduction. Also, due to temperature differences an energy exchange between the ions and electrons is established. The resulting exchange power density is represented by $\mathrm{P}_{\mathrm{ei}}$. For the species with the lowest temperature this exchange power density will be treated as a source; while, for the warmer species it is treated as a sink. Further assumptions implied in this model are that burnup fraction (typically $<1 \%$ ) can be ignored and that the background species are refueled with cold fuel. Also, the temperature and density of all species are assumed constant throughout the plasma. The power flows indicated in Fig. 3.1 can be incorporated into an overall balance as follows:

The amount of fusion power released in the plasma is:

$$
P_{f}=F P_{I},
$$

where by definition, $F$ is the fusion energy multiplication defined earlier in Eq. (2.1). In defining $\mathrm{P}_{f}$ in this fashion it is assumed that beam-plasma fusion dominates which is reasonable for the relatively low background temperatures of interest in early TCT experiments, which are explored here.*

The fraction of the fusion power which goes into the ions is given by :

\footnotetext{
* Recent studies ${ }^{(17)}$ have pointed out that beam-beam interactions will contribute some to the fusion, but this is neglected here. However, the low background temperature does insure that fusion between target ions, i.e. "Maxwellian" fusion, is indeed negligible.
} 


$$
P_{f, i}=\frac{P_{f}}{Q_{f}} \sum_{j=1}^{N} \frac{E_{j}}{Q_{f}} \int_{0}^{\tau_{S D}^{j}}\left\langle\frac{d E}{d t}\right\rangle_{i}^{j} d t
$$

where,

$$
\begin{aligned}
& Q_{f}=\text { the energy released per fusion, } \\
& E_{j}=\text { the initial energy of the } j^{\text {th }} \text { charged fusion product, } \\
& \left\langle\frac{d E}{d t}\right\rangle_{i}^{j}=\text { the background ion contribution to }\left\langle\frac{d E}{d t}\right\rangle \text { for the } j^{\text {th }} \\
& \tau_{S D}^{j}=\text { the slowing-down time (or containment time, if } \\
& \text { smaller) for the } j^{\text {th }} \text { charged fusion product. }
\end{aligned}
$$

To account for the possibility that fusion products can leak out before completely thermalizing, we define $\tau_{S D}^{j}$ as the smaller of the slowing down time or the fusion product containment time $\left(\tau_{p}^{i}\right)$.

Likewise the fraction of the fusion power which goes into the electrons is given by:

$$
P_{f, e}=\frac{P_{f}}{Q_{f}} \sum_{j=1}^{N} \frac{E_{j}}{Q_{f}}: \int_{0}^{\tau_{S D}^{j}}\left\langle\frac{d E}{d t}\right\rangle_{e}^{j} d t .
$$

The injection power is given by:

$$
P_{I}=\frac{n_{b} E_{I}}{\tau_{b}}
$$

where,

$$
\begin{aligned}
& n_{b}=\text { the number of beam ions per unit volume, } \\
& \tau_{b}=\text { the effective particle containment time for beam ions. }
\end{aligned}
$$

The fraction of the injected power going to the ions is found from a treatment analogous to that for fusion products, i.e., 


$$
P_{I, i}=\frac{P_{I}}{E_{I}} \int_{0}^{\tau_{S D}^{b}}\left\langle\frac{d E}{d t}\right\rangle_{i}^{b} d t
$$

where,

$$
\begin{aligned}
\left\langle\frac{d E}{d t}\right\rangle_{i}^{b}= & \text { the background ion contribution to }\left\langle\frac{d E}{d t}\right\rangle \text { for the beam } \\
& \text { ion, } \\
\tau_{S D}^{b}= & \text { the slowing-down time for the beam ion (note that if } \\
& \left.\tau_{b}<\tau_{S D}^{b} \text { then } \tau_{S D}^{b} \equiv \tau_{b}\right) .
\end{aligned}
$$

Similarly the fraction of injected power which goes to the electrons is

$$
P_{I, e}=\frac{P_{I}}{E_{I}} \int_{0}^{\tau_{S D}^{b}}\left\langle\frac{d E}{d t}\right\rangle_{e}^{b} d t .
$$

The power which is lost from the ions due to leakage and thermal conduction is defined as:

$$
P_{\ell, i}=\frac{3}{2} \frac{n_{i} T_{i}}{\tau_{E, i}},
$$

while that from the electrons is:

$$
P_{\ell, e}=\frac{3}{2} \frac{{ }^{n} e^{T} e}{\tau_{E, e}} .
$$

Here $\tau_{E, i}$ and $\tau_{E, e}$ represent the energy confinement times for the background ions and electrons, respectively.

The radiation power which predominately comes from the electrons can be divided into Bremsstrahlung $\left(\mathrm{P}_{\mathrm{Br}}\right)$ and cyclotron $\left(\mathrm{P}_{\mathrm{Cy}}\right)$ emission. The Bremsstrahlung power ${ }^{(18)}$ is given by: 


$$
\mathrm{P}_{\mathrm{Br}}\left(\mathrm{keV} / \mathrm{sec} / \mathrm{cm}^{3}\right)=3.34 \times 10^{-15} \mathrm{n}_{\mathrm{e}} \mathrm{T}_{\mathrm{e}}^{1 / 2} \sum_{j} \mathrm{n}_{j} \mathrm{z}_{j}^{2}
$$

where the sum over $\mathrm{j}$ includes all background ion species and the units. employed are: $n_{e}$ and $n_{j}$ in $\mathrm{cm}^{-3}, T_{e}$ in $\mathrm{keV}$.

Cyclotron emission is more complicated to handle because reflection from metallic walls and reabsorption in the plasma can significantly reduce losses. This is a difficult process to accurately evaluate, particularly, when the possibility that radiation damage to the wall which may affect reflection, is taken into account (19). The cyclotron expression (20) used in this work is given by:

$$
\mathrm{P}_{\mathrm{Cy}}\left(\mathrm{keV} / \mathrm{sec} / \mathrm{cm}^{3}\right)=1.56 \times 10^{-16} \frac{(1-\beta)}{\beta} \mathrm{dn}^{2} \mathrm{~T}_{\mathrm{e}}^{2} \mathrm{~K}_{\mathrm{c}}
$$

where,

$$
\begin{aligned}
& d=f^{2}\left(1+\frac{T_{i}}{f T_{e}}\right)\left(1+\frac{T_{e}}{204}\right) \\
& f=\frac{1}{n} \sum_{j} n_{j} z_{j}=\frac{n_{e}}{n}
\end{aligned}
$$

and densities are in $\mathrm{cm}^{-3}$ and temperatures in keV. The sum over $j$ includes all background ion species and the total ion number density. is given by $n$. Also note that here all ions are assumed to be at the same temperature $T_{i}$. As usual, the ratio of the plasma kinetic pressure to the magnetic pressure is defined as $B$. (Also see Eq. 3.26).

The dimensionless factor $K_{c}$ (the "cyclotron coefficient") represents the fraction of the cyclotron radiation which is ultimately absorbed by the first wall. 
The expression for $K_{c}$, used in this work, was obtained from T. Chu ${ }^{(21)}$ and is given by:

$$
K_{c}=K_{c}^{\prime}+\left(K_{L}-K_{c}^{\prime}\right) f
$$

where $f$ is the hole fraction of the reflecting wall and $K_{c}^{\prime}$ represents the value of the cyclotron coefficient if the wall had no holes while $\mathrm{K}_{\mathrm{L}}$ represents the limit where the wall reflectivity is zero.

A form of $K_{c}^{\prime}$ given by Krajcik ${ }^{(22)}$ for a cylindrical plasma, which was used in this work, is:

$$
K_{c}^{\prime}=e^{b_{0}} T_{e}{ }^{\left(b_{1}+b_{2} \ln \left(T_{e}\right)\right)}
$$

Where,

$$
\begin{aligned}
& b_{0}=-7.285-6.481 \times 10^{-2} n-4.582 \times 10^{-1} n^{2} \\
& +5.356 \times 10^{-2} n^{3}-2.435 \times 10^{-3} n^{4} \text {, } \\
& b_{1}=1.804-6.029 \times 10^{-1} \eta+1.704 \times 10^{-1} \eta^{2} \\
& -2.077 \times 10^{-2} n^{3}+9.599 \times 10^{-4} n^{4} \text {, } \\
& b_{2}=-9.718 \times 10^{-2}+1.070 \times 10^{-1} \eta-1.929 \times 10^{-2} \eta^{2} \\
& +2.122 \times 10^{-3} n^{3}-9.561 \times 10^{-5} n^{4} \text {, } \\
& \eta=\log _{10}\left(\frac{d n_{e}}{B(B \rho)^{1 / 2}}\right)-19 \\
& B=\text { the magnetic field (Tesla) in the plasma (i.e. the } \\
& \text { vacuum field } \left.B_{v}=B / \sqrt{1-B}\right) \text {, } \\
& \mathrm{d}=\text { diameter of plasma in meters, } \\
& \rho=\text { resistivity of the first wall in ohm-m, }
\end{aligned}
$$




$$
\mathrm{n}_{\mathrm{e}}=\text { the electron number density in } \mathrm{m}^{-3} \text {. }
$$

The expression for $\mathrm{K}_{\mathrm{L}}$ given in Ref. 18 takes the form:

$$
K_{L}=\frac{2.1 \times 10^{-3} \mathrm{~T}_{\mathrm{e}}^{7 / 4}}{\mathrm{~L}^{1 / 2}}
$$

where the plasma depth, $L$, is defined as:

$$
L=\frac{d n_{e} e}{c B \varepsilon_{0}}
$$

Here $T_{e}$ is in keV, $d$ in $m, e$ in $C, c$ in $m / s e c, B$ in Tesla and $n_{e}$ in $m_{.}^{-3}$. The power which is exchanged between the background electrons and ions due to temperature differences is discussed in Ref. 13. A numerical form for this term is given by:

$$
P_{e i}=\frac{3 n_{e}\left(T_{e}-T_{i}\right)}{2 \tau_{e i}}
$$

with:

$$
\tau_{e i}=\frac{3 m_{e} m_{i}}{8 \sqrt{2 \pi} n_{i} \ln \Lambda z_{i}^{2} e^{4}}\left(\frac{T_{e}}{m_{e}}+\frac{T_{i}}{m_{i}}\right)^{3 / 2}
$$

For Eqs. (3.21) and (3.22) the cgs system of units has been used except for the temperatures which are in $\mathrm{keV}$. In this work it is assumed that $\ln \Lambda \approx 20$. Finally the overall balance equation for the ions can be written as:

$$
P_{f, i}+P_{I, i}+P_{e, i}=P_{l, i}
$$

while a corresponding balance for the electrons is:

$$
P_{f, e}+P_{I, e}=P_{e, i}=P_{\ell, e}+P_{\text {rad }}
$$


Method of Solution

It is clear that Eqs. (3.23) and (3.24) depend on the parameters $E_{I}, T_{e}$ and $T_{i}$ (all other factors will be treated as constants for a specific case). This then leaves two equations with three unknowns for a given case. In order to determine the relationships between the above parameters the following scheme is used. First a value of $E_{I}$ is chosen. Then the background ion and electron temperatures are evaluated. Next a different $E_{I}$ is chosen and the above process is repeated, allowing construction of plots of $\mathrm{T}_{i}$ and $\mathrm{T}_{e}$.vs. $\mathrm{E}_{\mathrm{I}}$. A more detailed outline of this process is shown in Fig. 3.2. The system parameters which must be initialized are:

$\begin{array}{ll}\tau_{b} & - \text { particle containment time of the beam ions, } \\ \tau_{E, i(e)} & \text { - energy confinement time for the background ions } \\ & \quad(\text { electrons), } \\ B_{v} & - \text { vacuum magnetic field, } \\ d & - \text { plasma diameter, } \\ p & - \text { resistivity of the first wall, } \\ f & - \text { fractional hole area of the first wall, } \\ j_{I} & - \text { the injection rate per volume of plasma for beam } \\ & \text { ions, }\end{array}$

In this work the particle containment time for the charged fusion products $\left(\tau_{p}^{j}\right)$ is assumed to be the same as the beam containment time, $\tau_{b}$. Also the energy confinement times $\tau_{E, i}$ and $\tau_{E, e}$ are taken to be equal and subsequently, they are referred to as $\tau_{E}$.

Numerical values for these system parameters are given in Table 3.1 for both a $D \rightarrow T$ and a $D \rightarrow{ }^{3}$ He TCT systems. The $D \rightarrow T$ values are based 


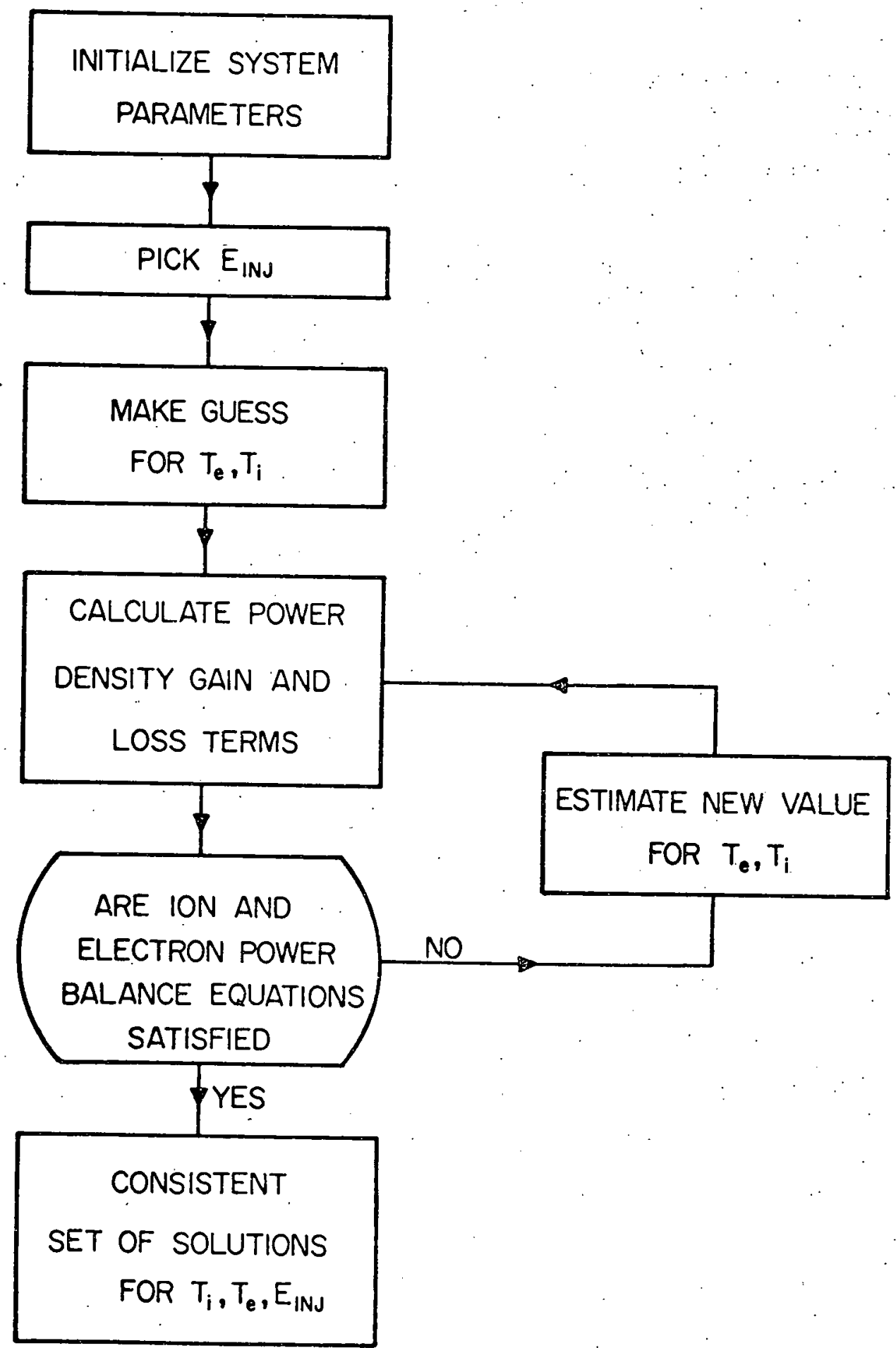

Fig. 3.2 Flow diagram showing the calculational technique. 
Table 3.1 System Parameters.

\begin{tabular}{|c|c|c|}
\hline Quantity & $\mathrm{D} \rightarrow \mathrm{T}$ & $\mathrm{D} \rightarrow{ }^{3} \mathrm{He}$ \\
\hline Plasma Radius, d & $56 \mathrm{~cm}$ & $45 \mathrm{~cm}$ \\
\hline Vacuum Magnetic Field, $\mathrm{B}_{v}$ & $49 \mathrm{kG}$ & $98 \mathrm{kG}$ \\
\hline Energy Confinement Time, $\tau_{E}$ & $.2 \mathrm{sec}$ & $.15 \mathrm{sec}$ \\
\hline Beam Confinement Time, $\tau_{b}$ & $.2 \mathrm{sec}$ & $.15 \mathrm{sec}$ \\
\hline Target Ion Density, $n_{i}$ & $5.6 \times 10^{13} / \mathrm{cm}^{3}$ & $3.4 \times 10^{13} / \mathrm{cm}^{3}$ \\
\hline Injection Energy, $E_{I}$ & $150 \mathrm{keV}$ & $300 \mathrm{keV}$ \\
\hline Injection Rate/Volume, $j_{I}$ & $2.0 \times 10^{13} /\left(\mathrm{cm}^{3} \mathrm{sec}\right)$ & $1.6 \times 10^{13} /\left(\mathrm{cm}^{3} \mathrm{sec}\right)$ \\
\hline Plasma Volume, V & $1.55 \times 10^{7} \mathrm{~cm}^{3}$ & $1.14 \times 10^{7} \mathrm{~cm}^{3}$ \\
\hline Resistivity of First Wall, $\rho$ & $9.7 \times 10^{-7} \Omega-\mathrm{m}$ & $9.7 \times 10^{-7} \Omega-\mathrm{m}$ \\
\hline First Wall Hole Fraction, $f$ & .1 & .1 \\
\hline Effective $Z$ of plasma, $Z_{\text {eff }}$ & 1 & 2 \\
\hline
\end{tabular}


on Ref. 2 while the $D \rightarrow{ }^{3}$ He values were projected from the $D \rightarrow T$ values. The $\mathrm{D} \rightarrow{ }^{3} \mathrm{He}$ calculations are included here since such experiments are planned prior to the ultimate $\mathrm{D} \rightarrow \mathrm{T}$ studied in TCT. Some of the results presented here were used in calculations by Petrie and Miley (23) to investigate the feasibility of using $D \rightarrow{ }^{3}$ He to simulate $D \rightarrow T$ fusion burns.

\section{The Pressure Balance}

In this model the number densities of each of the species is held constant by the introduction of new (cold) fuel at the exact rate at which the warmer fuel leaves the plasma. Since in this model the number densities are constant and the resulting background temperatures are found as a function of the injection energy, $\beta$ must vary in order to maintain a pressure balance. In this manner $\beta$ is defined as:

$$
B=\frac{\sum_{j} n_{j} k T_{j}+P_{b}}{B_{v}^{2} / 8 \pi},
$$

where the cgs system of units has been used and the sum over $j$ includes all background species, $P_{b}$ is the resulting average beam pressure, and $\mathrm{B}_{v}^{2} / 8 \pi$ is the vacuum magnetic pressure. From elementary Tokamak MHD theory $(24)$ it is predicted that $\beta$ would be limited to .037 and .027 for $D \rightarrow T$ and $D \rightarrow{ }^{3} \mathrm{He}$, respectively. Equation (3.25) can be written as:

$$
B=\frac{\left(\sum_{j} n_{j} k T_{j}\right)(1+3 \Gamma / 2)}{B_{v}^{2} / 8 \pi}
$$

Here $\Gamma$ is defined as the ratio of the average beam energy density to plasma energy density, i.e.: 


$$
\Gamma=\frac{\int_{E_{T h}}^{E_{T}} \eta_{b}(E) E d E}{3 / 2 \sum_{j=1}^{N} n_{j}{ }^{k T} j},
$$

where $\eta_{b}(E)$ is the number of beam particles in an energy interval, $\mathrm{dE}$, centered about $\mathrm{E}$ per volume of plasma. For steady state, assuming no sources or losses as the beam species slow down, then:

$$
\eta_{b}(E)=\eta_{b}\left(E_{I}\right)\left\langle\frac{d E}{d t}\right\rangle_{E_{I}} /\left\langle\frac{d E}{d t}\right\rangle_{E}
$$

However $\eta_{b}\left(E_{I}\right)\left\langle\frac{d E}{d t}\right\rangle_{E_{I}}$ is just the injection rate per volume of plasma and is denoted as $j_{I}$. Hence Eq. (3.27), can be rewritten as:

$$
\Gamma=\frac{j_{I} \int_{E_{T h}}^{E_{T}} \frac{E}{\left\langle\frac{d E}{d t}\right\rangle_{E}} d E}{3 / 2 \sum_{j=1}^{N} n_{j} k T_{j}} .
$$

$\underline{\text { Results }}$

In this section TCT performance is first based on the design parameters of Table 3.1 as a function of injection energy $E_{I}$, but the injection rate is arbitrarily maintained at the constant value of $2 \times 10^{13} /\left(\mathrm{cm}^{3} \mathrm{sec}\right)$. After that, the effect of varying various other key parameters is briefly examined.

In all of the subsequent cases presented it is found that the dominate heating mechanism is the injection power while the dominate loss is due to leakage $\left(P_{\ell, i}\right.$ and $\left.P_{\ell, e}\right)$. It was further found that the 
majority of the injection power went into the ions while the majority of the power associated with the fusion charged products went to the electrons. The radiation power loss ( $\mathrm{P}_{\mathrm{rad}}$ ) is generally small compared to $P_{\ell, e}$, and the Bremsstrahlung radiation is noticeably more significant than cyclotron emission.

\section{Performance With Design Parameters}

Based on these balances, F-values are given in Figs. 3.3 and 3.4 for deuterium injected into pure tritium and helium-3 respectively, as a. function of the injected energy. The results in these figures are considerably different than the ones presented earlier in Chapter 2 since now the background ion and electron temperatures vary with the injection energy as shown in Figs. 3.5 and 3.6. In general, TCT operation has been based on a "design" value of $\mathrm{T}_{\mathrm{e}} \sim 5 \mathrm{keV}$ with $\mathrm{T}_{i} \sim 0$ (cold background ion approximation) for deuterium injected into a pure tritium background. With the standard parameters of Table 3.1 , this requires an injection energy of $\sim 195 \mathrm{keV}$ (see Fig. 3.5). For the same injection rate, but lower injection energies, Fig. 3.5 shows that, as might be expected, $\mathrm{T}_{\mathrm{e}}$ decreases. The net result is that, as seen from the F-value plot in Fig. 3.3, the energy multiplication also decreases relative to the design $\left(\mathrm{T}_{\mathrm{e}}=5 \mathrm{keV}\right)$ curve.

Similar effects are observed for deuterium injected into a pure helium-3 target however, since the $D \rightarrow{ }^{3}$ He fusion reactivity for injection energies between $100-200 \mathrm{keV}$, is $\approx 1000$ times smaller than $\mathrm{D} \rightarrow \mathrm{T}$. (see Figs. 1.1 and 1.2 ) The F-values are also $\sim 1000$ times smaller than $D \rightarrow T$. In fact, at $E_{I}=160 \mathrm{keV}$ the $F$-value ratio between $D \rightarrow{ }^{3} \mathrm{He}$ and $\mathrm{D} \rightarrow \mathrm{T}$ is $1.8 \times 10^{-3}$. 


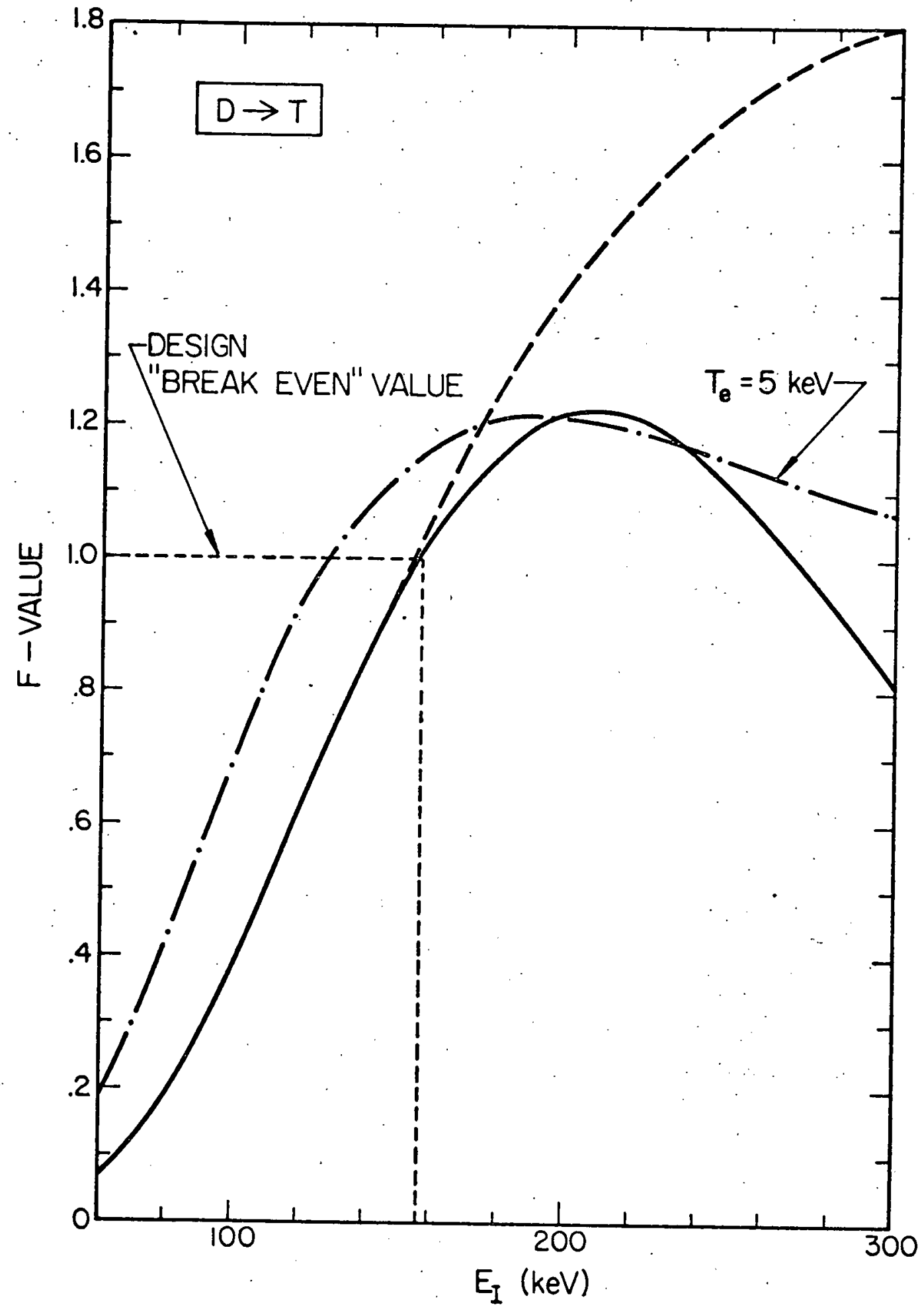

Fig. 3.3 F-Values for deuterium injected into a pure tritium target with self-consistent background temperatures for the parameters of Table 3.1 (solid line). The dashed line assumes an infinite containment time for the beam ions (all other parameters are the same as in Table 3.1) and the broken line shows $F$ for the "design" value of $\mathrm{T}_{\mathrm{e}}=5 \mathrm{keV}$ with $\mathrm{T}_{i} \sim 0$. It is observed that according to this model, the "breakeven" $\mathrm{E}_{\mathrm{I}}$ occurs at $\sim 158 \mathrm{keV}$. 


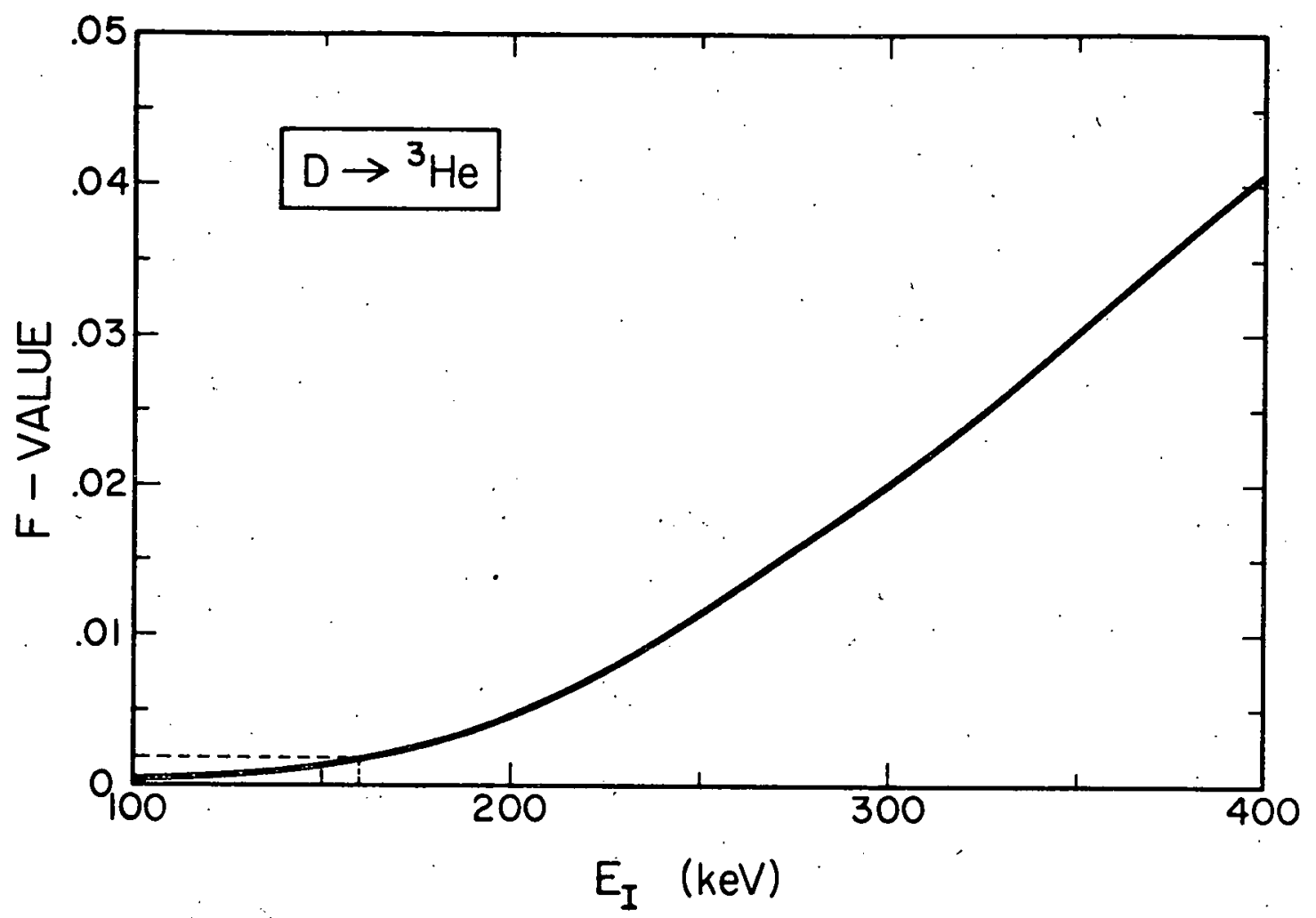

Fig. 3.4 F-Values for deuterium injected into a pure helium-3 target with self-consistent background temperatures for the parameters of Table 3.1. 


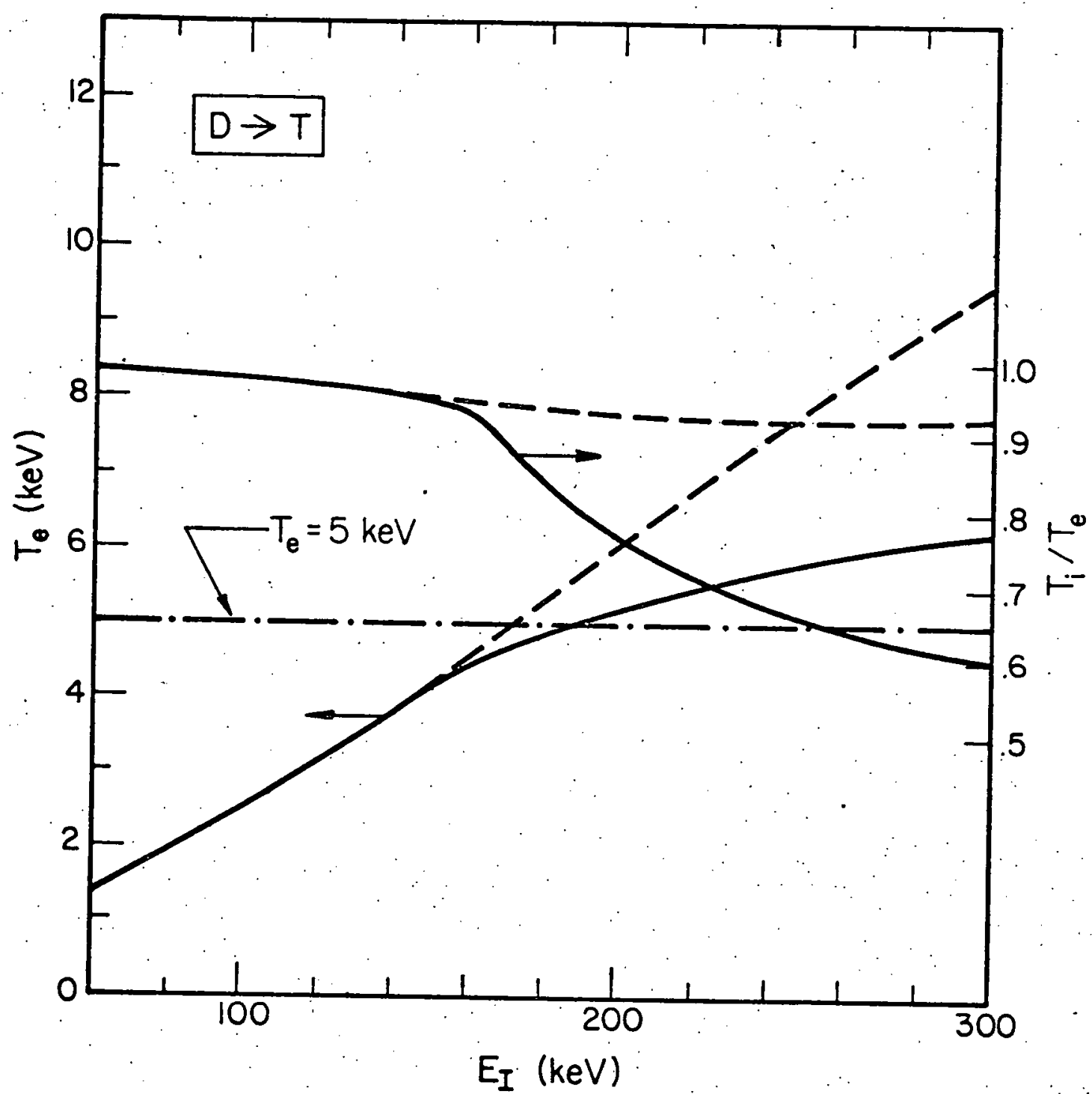

Fig. 3.5 Temperature variations for deuterium injected into a pure tritium target using parameters given in Table 3.1. The dashed line assumes an infinite containment time for the beam ions (all other parameters are the same as in Table 3.1). 


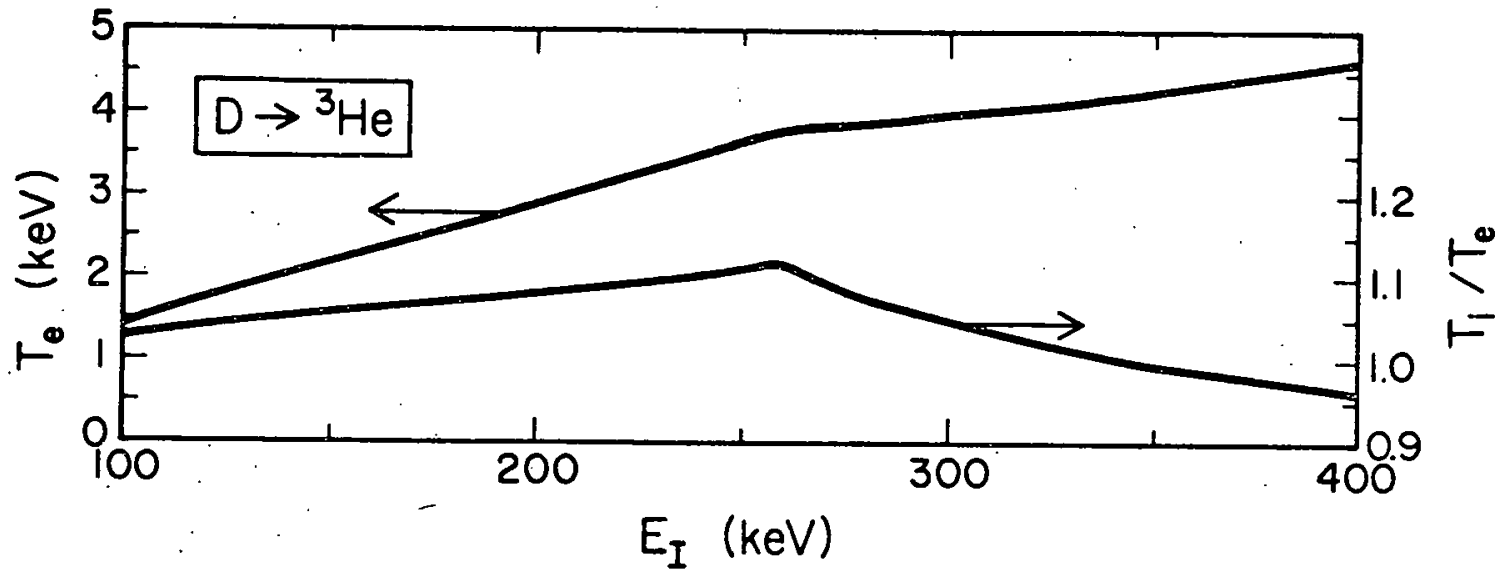

Fig. 3.6 Temperature variation for deuterium injected into a pure helium-3 target defined by the parameters of Table 3.1 . 
The marked decrease in the plots of $\mathrm{T}_{i} / \mathrm{T}_{\mathrm{e}}$ (at $\mathrm{E}_{\mathrm{I}} \approx 160 \mathrm{keV}$ for $D \rightarrow T$ and $E_{I} \approx 260 \mathrm{keV}$ for $D \rightarrow{ }^{3} \mathrm{He}$ ) is a result of the slowing down time, $\tau_{\text {SD }}$, being limited by the beam containment time, $\tau_{b}$. The slowing down time is shown in Fig. 3.7 as a function of the injection energy (and hence $\mathrm{T}_{e}$ and $\mathrm{T}_{i}$ ) for both $\mathrm{D} \rightarrow \mathrm{T}$ and $\mathrm{D} \rightarrow{ }^{3} \mathrm{He}$. In this model the beam containment time was assumed to be a constant (see Fig. 3.7). In reality this will be a function of the background temperatures and hence of the injection energy. The effect of $\tau_{\mathrm{SD}}$ being limited by $\tau_{\mathrm{b}}$ could still exist depending on the drift velocity (out of the plasma) of the injected ion and its slowing down time.

A critical injection energy, $E_{c r}$ ' is reached when $\tau_{S D}=\tau_{b}$ and for energies greater than this, the injected species leave the plasma before transferring all of their energy to the target plasma. Since most of the energy transferred at the lower injected ion energies goes into the background ions, the ion temperature is drastically reduced by the premature ion escape. The gain in electron temperature expected due to the higher injection energy is also reduced somewhat, simply because the total fraction of the injected energy is reduced.

The reduction in temperature rise with increasing injection energy has the net effect that increasing $E_{I}$ above $\sim 180 \mathrm{keV}$ for $D \rightarrow T$ is not as effective in increasing $F$ as might have been expected otherwise. This point is illustrated by the "extrapolated" F-value curve in Fig. 3.3 which represents the case where all injected ions are contained $\left(\tau_{b}\right.$ is infinite).

Plots of the $\beta$ and $\Gamma$ values for the above $D \rightarrow T$ and $D \rightarrow{ }^{3} \mathrm{He}$ cases are shown in Figs. 3.8 and 3.9. As shown in Fig. 3.8 the $\beta$-values lie well below the stability limits of .037 and .027 for $D \rightarrow T$ and $D \rightarrow{ }^{3} \mathrm{He}$ respec- 


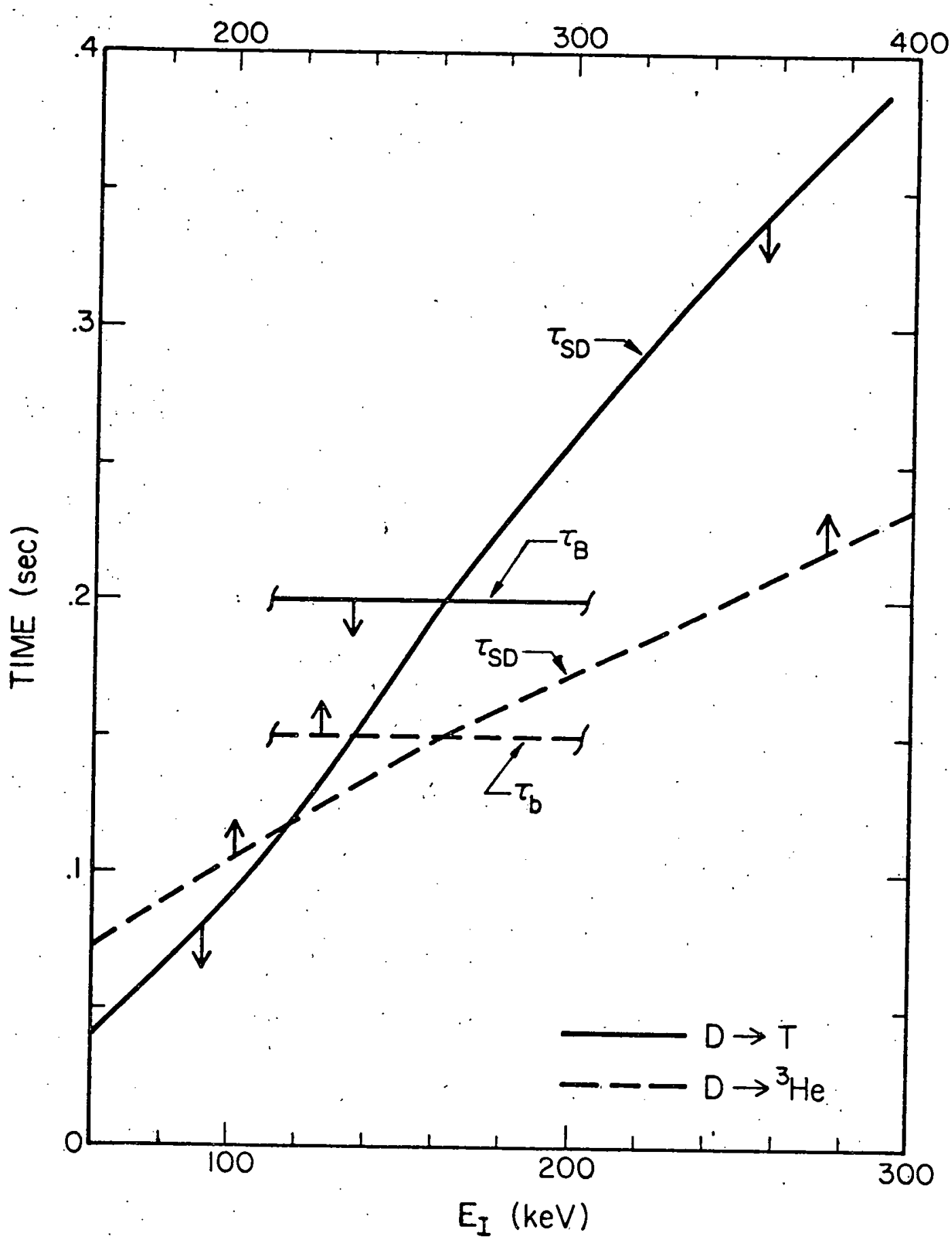

Fig. 3.7 Slowing down and beam containment time as a function of the injected energy for both $D \rightarrow T$ and $\mathrm{D} \rightarrow 3 \mathrm{He}$ using system parameters as given in Table 3.1. 
55

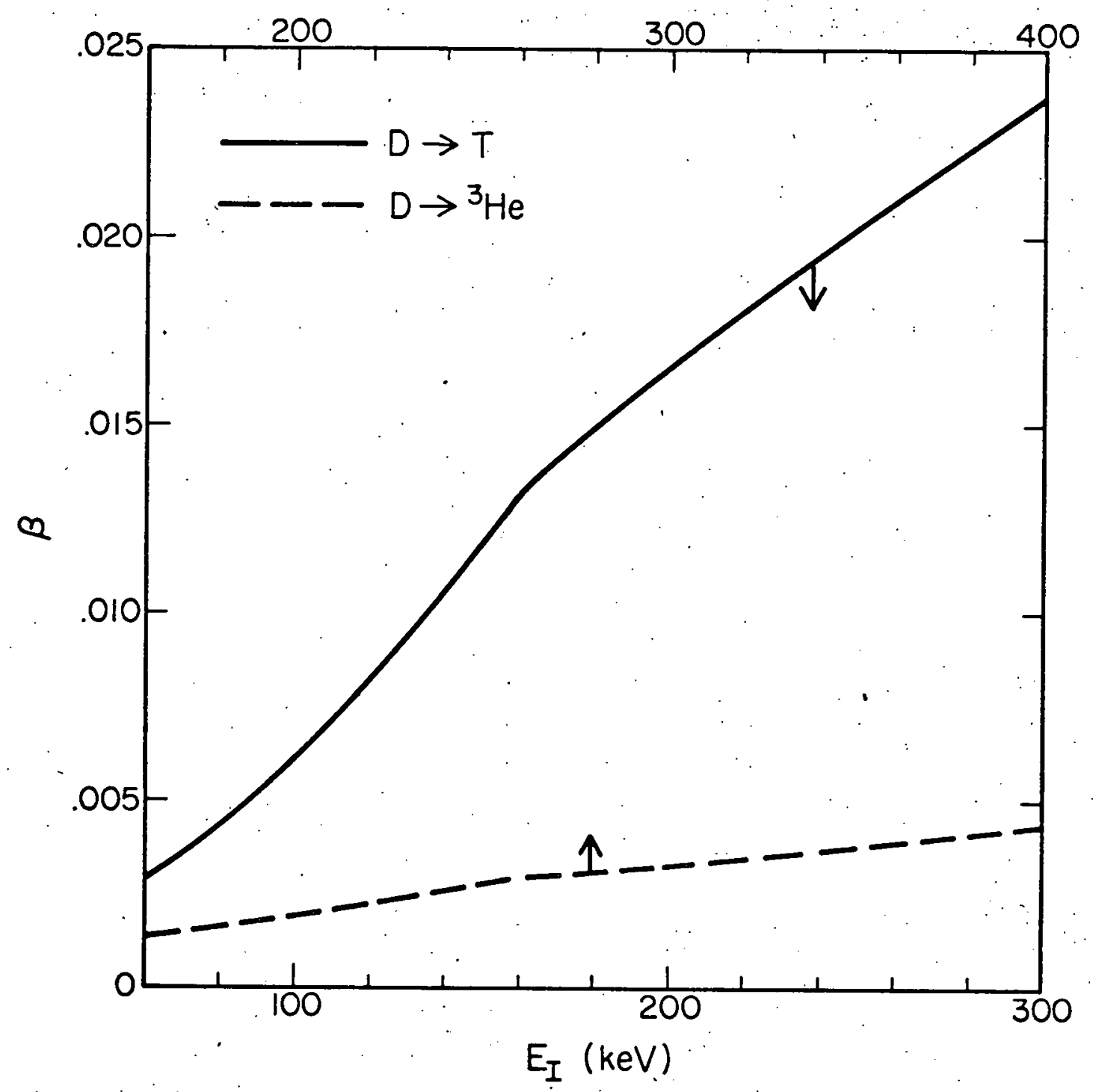

Fig. $3.8 \quad \beta$ values as a function of injection energy for both $D \rightarrow T$ and $D \rightarrow 3^{3} \mathrm{He}$ using system parameters as given in Table 3.1 . 


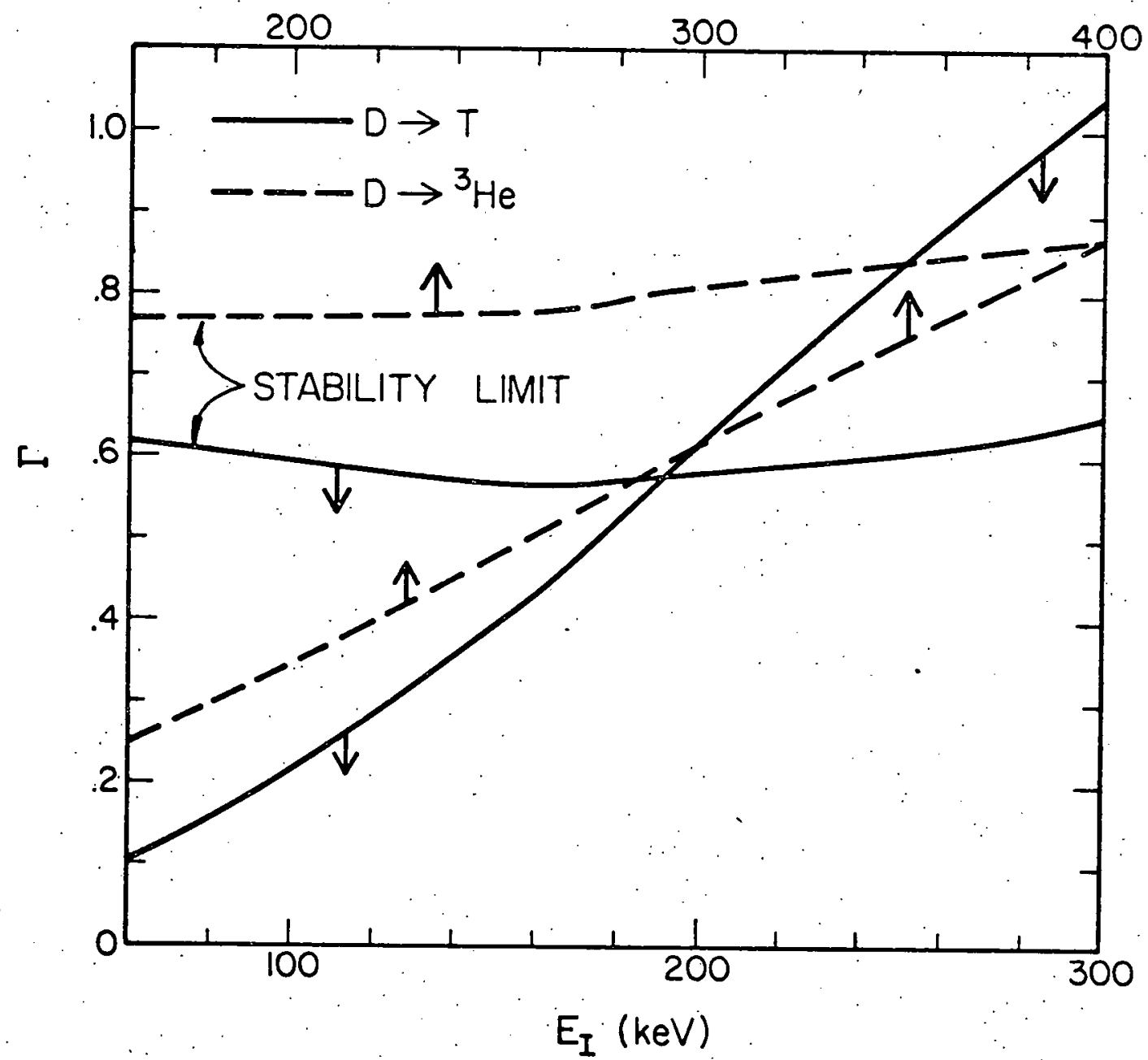

Fig. 3.9 $\Gamma$-values as a function of injection energy for both $D \rightarrow T$ and $D \rightarrow 3$ He using system parameters as given in Table 3.1. 
tively. The stability limit for $\Gamma$ is shown in Fig. 3.9, based upon Ref. 25. From this figure it is found that the $D \rightarrow T \Gamma$-values exceed the stability limit for $E_{I}>190 \mathrm{keV}$, while for $\mathrm{D} \rightarrow{ }^{3} \mathrm{He}$ the stability limit is reached for $E_{I}>400 \mathrm{keV}$.

\section{Effect of Parameter Variations}

The effects of four key parameters in TCT performance have been considered, namely: energy confinement time $\tau_{E}$; beam containment time $\tau_{b}$; injector performance $\left(j_{I}, E_{I}\right)$; and impurity fraction.

\section{i) Energy confinement time}

The effect of the background energy confinement time $\tau_{E}$ on the energy multiplication values is shown in Figs. 3.10 and 3.11 . As $\tau_{E}$ increases, the leakage power due to particle loss and conduction decreases. Consequently, the background ion and electron temperatures increase which in turn increases the energy multiplication values. This is done by reducing the drag on the injected particles and increasing $\langle\sigma v\rangle_{b}$ (recall Figs. 1.1-1.6). However, for injection energies in the range of 200 to $300 \mathrm{keV}$, the various curves in Fig. 3.10 cross over, so that larger confinement times actually result in lower F-values. This cross over effect is caused by the competing effects of increased background temperatures and the limitation of the slowing down time, $\tau_{S D}$ : The increase in the background temperatures have a positive effect on the F-values (recall Figs. 2.2-2.3) however, the increase in the background temperatures also increases the slowing down time, $\tau_{S D}$, until it matches the beam containment time, $\tau_{b}$. This limitation on $\tau_{S D}$ has a negative effect on the $F$-value because the range of in-. tegration in Eq. (2.4) is reduced. As a net result the limitation on 


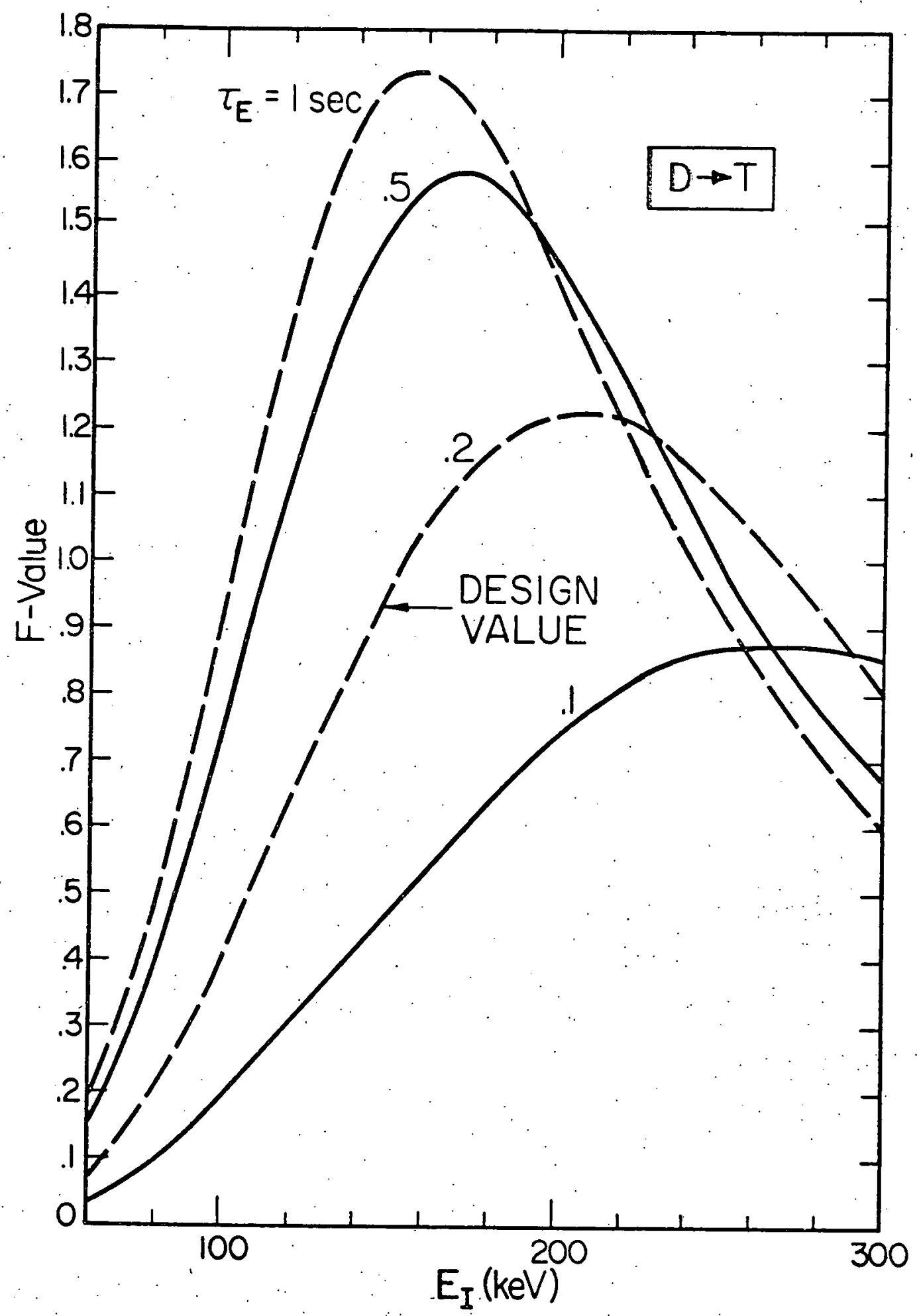

Fig. 3.10 Effect of energy confinement time on F-Values for deuterium injected into a pure tritium background. 


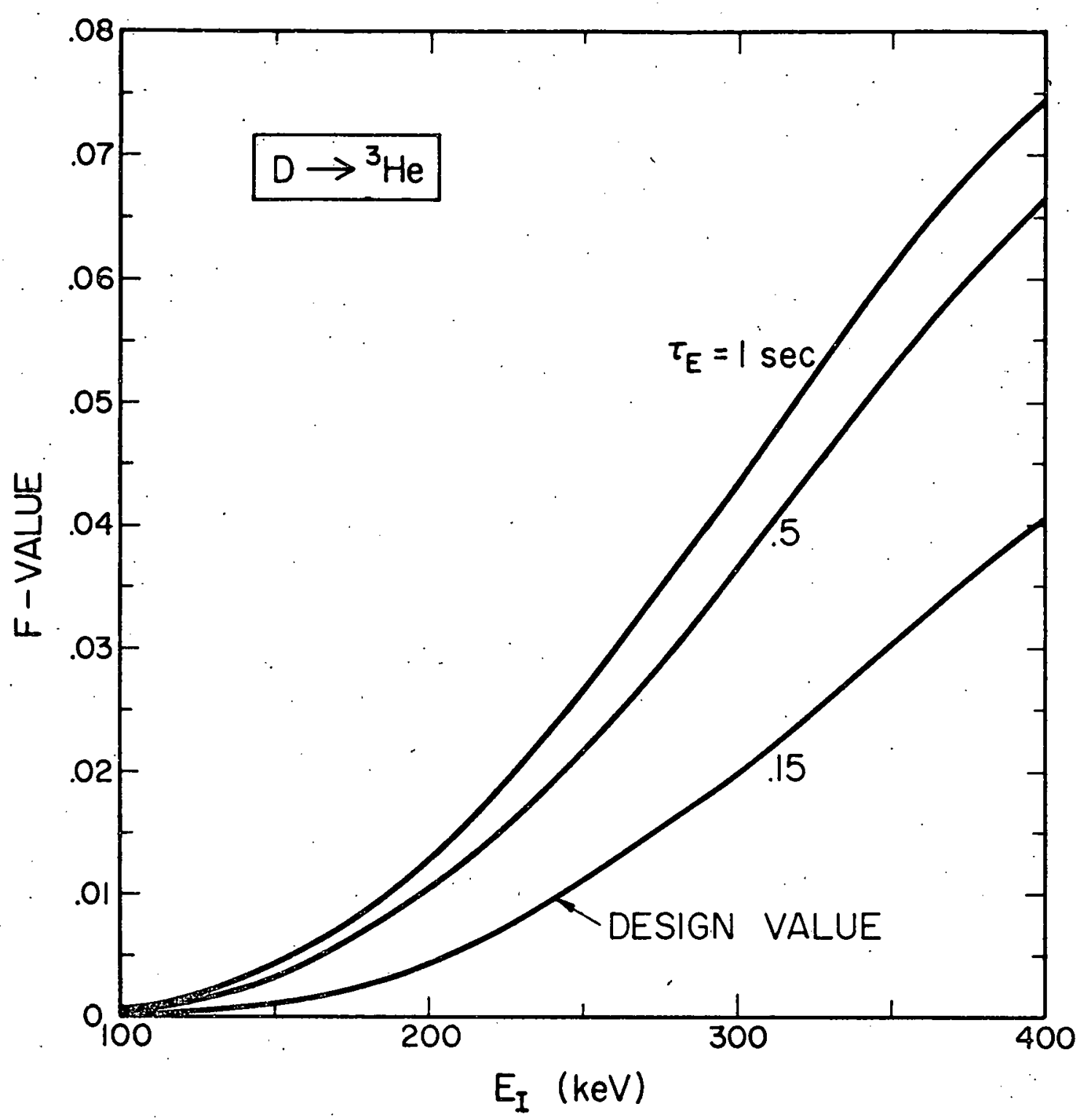

Fig. 3.11. Effect of energy confinement time on F-values for deuterium injected into a pure helium-3 background. 
$\tau_{\text {SD }}$ outweighs the temperature effects resulting in the cross over. It should be noted that for the $D \rightarrow{ }^{3}$ He case the dominate effect is the increased background temperatures.

The curves shown in Figs. 3.10 and 3.11 are for a constant value of energy confinement time, $\tau_{E}$, independent of the target temperature, i.e. independent of the injection energy. In fact $\tau_{E}$ will vary somewhat making it necessary to interpolate between curves if a "realistic" variation is desired. This can be done with the aid of Fig. 3.12 in which $n_{e} \tau_{E}$ is plotted as a function of $E_{I}$ for $D \rightarrow T$. The values for $n_{e} \tau_{E}$ were obtained from a four regime model presented in Ref. 24 and is repeated below:

$$
\begin{aligned}
& \mathrm{n}_{\mathrm{e}} \tau_{E}=(1.2-2.9) \times 10^{14} \mathrm{I}^{2} \sqrt{T_{\mathrm{e}}} / \mathrm{Z}_{\mathrm{eff}} \text {, Pseudoclassic } \\
& n_{e} \tau_{E}=5.9 \times 10^{14} I^{4} b_{t}^{2} \beta_{p_{e}}^{2} Z_{\text {eff }} A^{3 / 2} / T_{e}^{11 / 2} \\
& \text { Trapped-Electron-I } \\
& n_{e} \tau_{E}=1.1 \times 10^{13} I^{2} \sqrt{T} e^{A} / Z_{e f f}, \quad \text { Trapped-Electron-II }
\end{aligned}
$$

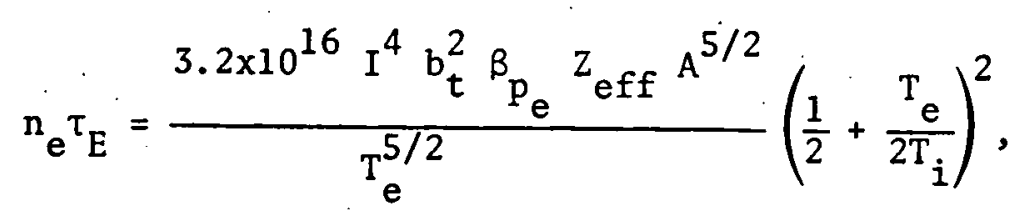

$$
\begin{aligned}
& \text { Trapped-Ion }
\end{aligned}
$$

where $\quad b_{T}=B / 50 k G$,

$$
\begin{aligned}
& A \quad=R /(3 a), \\
& B \quad=\text { Magnetic field strength (KG), } \\
& R \quad=\text { Major radius, } \\
& a \quad=\text { Plasma radius, } \\
& I \quad=\text { Toroidal current (MA), } \\
& Z_{\text {eff }}=\text { Effective } Z \text { of the plasma, }
\end{aligned}
$$




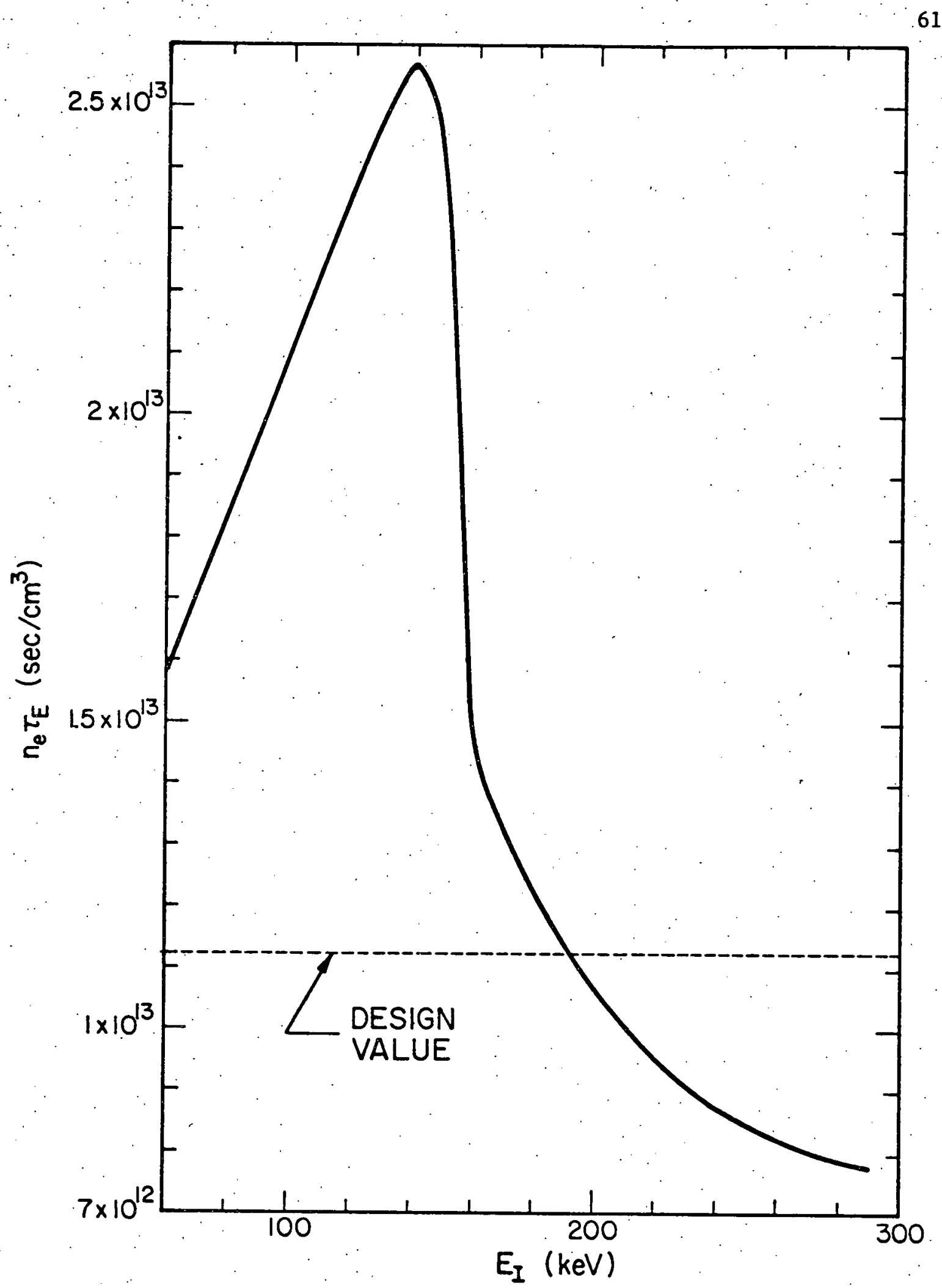

Fig. $3.12 \mathrm{n}^{\tau_{E}}$ values as a function of injection energy for for the $D \rightarrow T$ system parameter given in Table 3.1 . 


$$
\beta_{p_{e}}=\text { Poloidal electron } \beta
$$

It should be noted that Eq. (3.32) is just a lower collision frequency regime of Eq. (3.31). It is found that the plot in Fig. 3.12 starts off in the lower collision frequency Trapped-Electron regime (Eq. 3.32), but switches to the Trapped-Ion mode (Eq. 3.33) when $E_{I} \approx 140 \mathrm{keV}$.

\section{ii) Beam containment time}

The variation of the F-values due to the beam containment time, $\tau_{b}$, is demonstrated for $D \rightarrow T$ in Fig. 3.13. For low injection energies ( $<100 \mathrm{keV})$ and hence low background temperatures, the F-values are insensitive to $\tau_{b}$. However, as discussed earlier, when the slowing down time becomes equal to the containment time, the beam ions leave the plasma prematurely thus reducing the F-value. Hence, in order to attain the largest F-values we need $\tau_{S D} \sim \tau_{b}$.

\section{iii) Injector performance}

Up until now constant current injectors have been assumed (see Table 3.1 for the respective values). Two other cases of interest include constant power injectors and single stage injectors limited by voltage breakdown ${ }^{(26-29)}$ in which $j_{I^{\alpha}} E_{I}{ }^{-1}$ and $j_{I^{\alpha}} E_{I}^{-5 / 2}$ respectively.* A graph of the injected power density as a function of injection energy is given in Fig. 3.14 for the above three modes. In this figure mode 1 represents the constant current case, mode 2 the constant power case and mode 3 the voltage breakdown case. The latter two cases were normalized to the first case at $E_{I}=200 \mathrm{keV}$. In this

\footnotetext{
It is now thought that the TFTR will employ two stage injectors which are not considered here.
} 


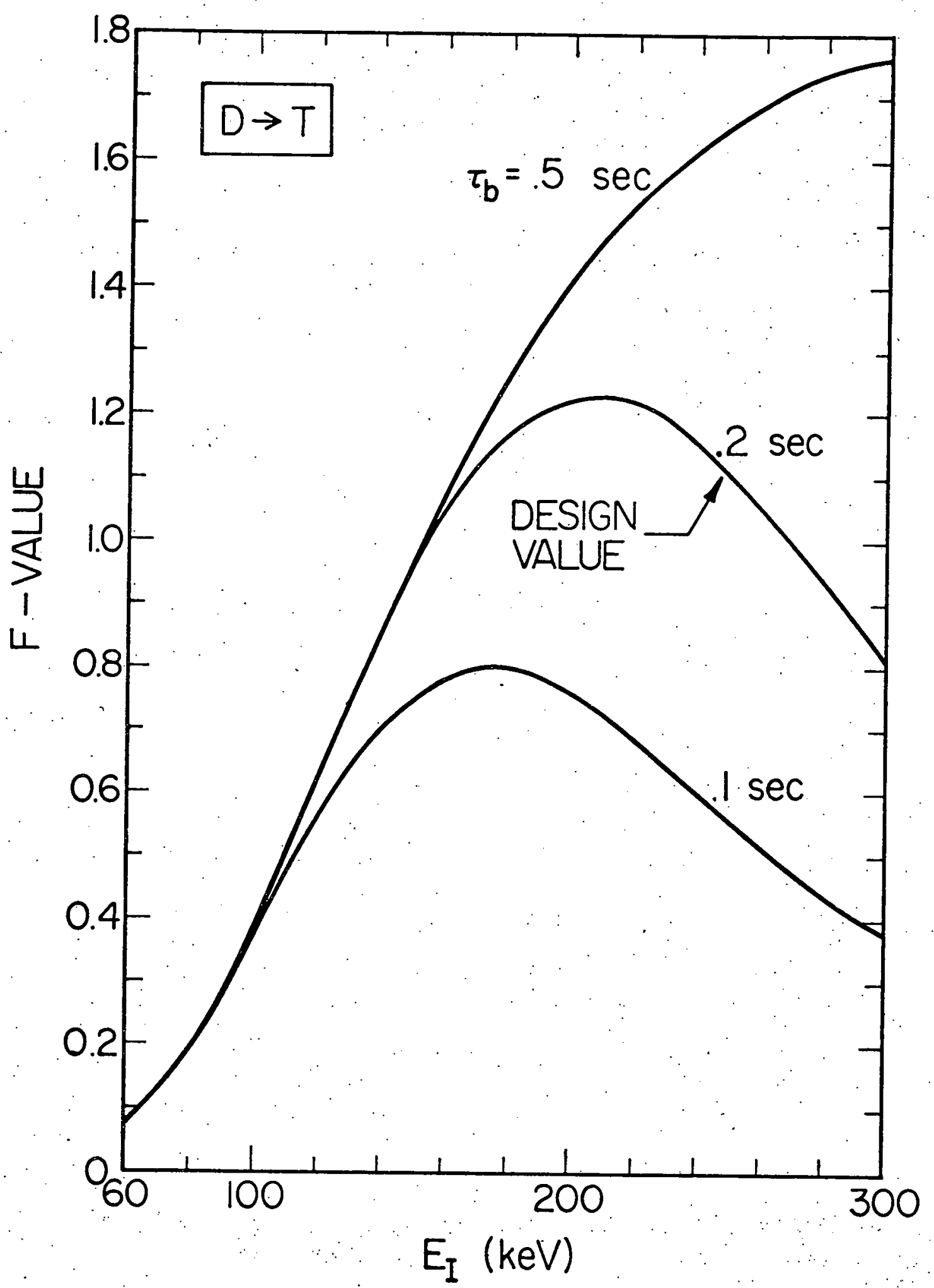

Fig. 3.13 Effect of the beam confinement times on F-Values for deuterium injected into a pure tritium target. 


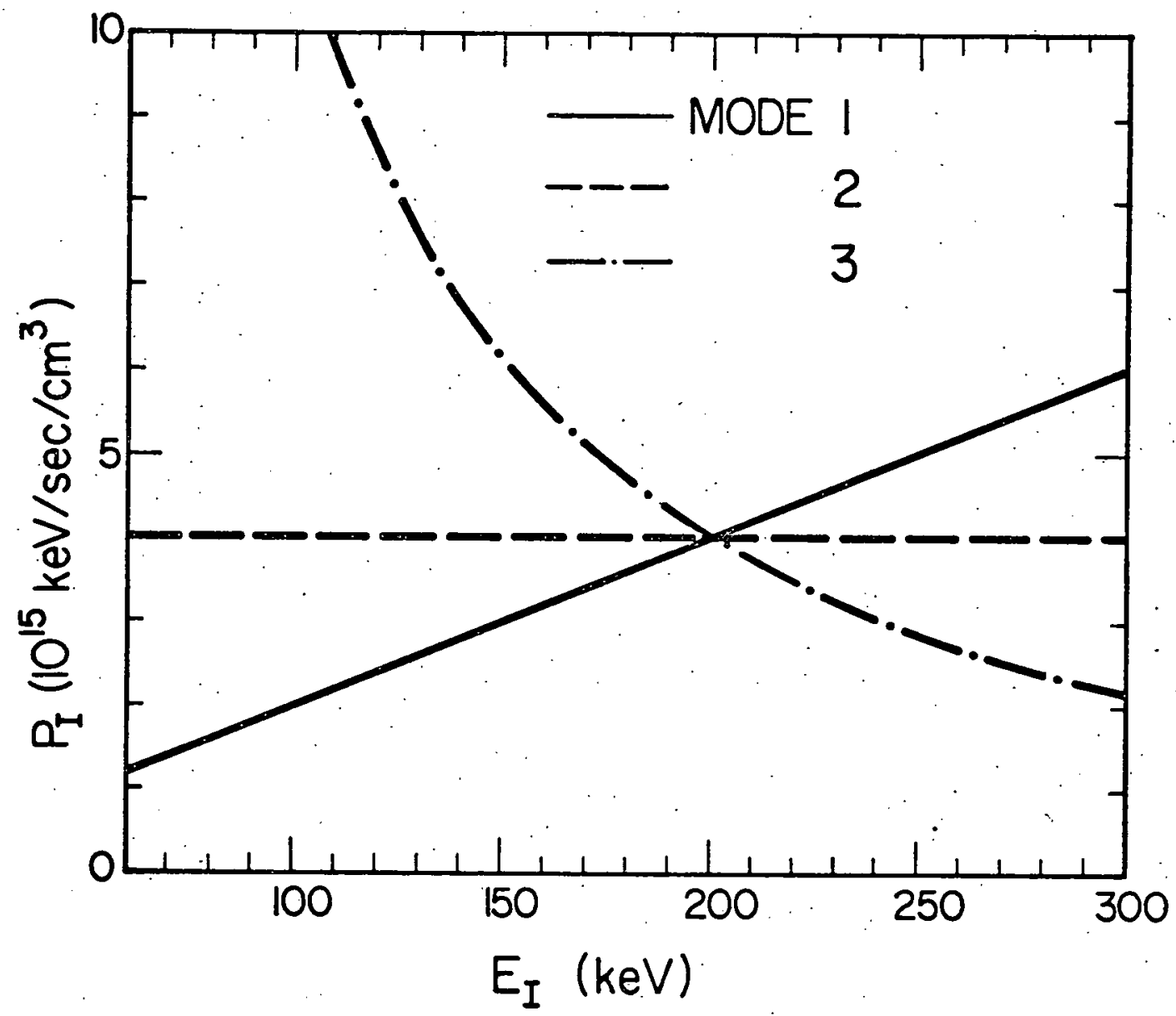

Fig. 3.14 Injection power for the 3 modes as a function of the injection energy. 
graph it should be noted that there is an upper bound on the power which one can actually achieve due to power supply limitations. To demonstrate the effects which the above three modes have on energy multiplication values, deuterium injected into a pure tritium target has been selected and is illustrated in Fig. 3.15. In general this figure shows that the F-values scale directly with the injection power, that is, the mode with the highest injection power also has the highest background temperatures which results in a higher F-value. The cross over between modes 1 and 2 near $275 \mathrm{keV}$ is the result of the limitation of $\tau_{S D}$ for the beam species as discussed earlier.

iv) Impurities

Until now all the results in this chapter have assumed a pure target background plasma. Now the effect on the energy multiplication value of an iron impurity is examined for $\mathrm{D} \rightarrow \mathrm{T}$.

As discussed in Ref. 30 values of $\mathrm{n}_{\mathrm{Fe}} / \mathrm{n}_{e}$ are expected to be in the range of $10^{-2}-10^{-3}$ where, $n_{F e}$ is the number density of the iron impurity (assumed fully stripped of electrons). In Fig. 3.16 the effect which the iron impurities have on the energy multiplication factors is given for the above concentrations. In this case the electron number density has been held to the design value of $5.6 \times 10^{13} / \mathrm{cm}^{3}$ while the background ion density $n_{i}$ was varied according to:

$$
n_{i}=n_{e}\left(1-26 n_{F e} / n_{e}\right)
$$

The effective $Z$ of the plasma which is defined by:

$$
z_{\text {eff }}=\sum_{j} \frac{n_{j} z_{j}^{2}}{n_{e}}
$$




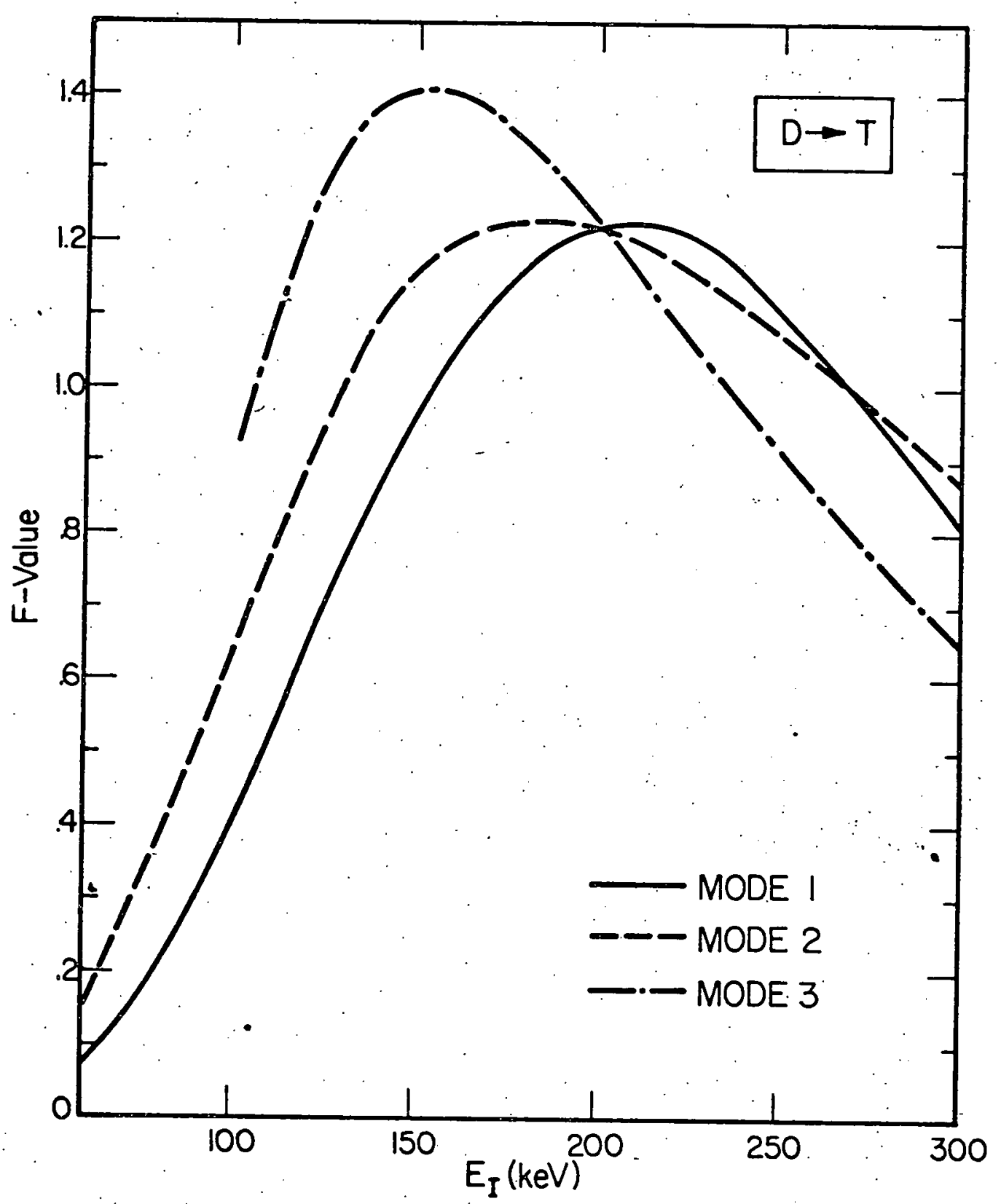

Fig. 3.15 Variation of F-values due to the three injection modes for deuterium injected into a pure tritium background. 


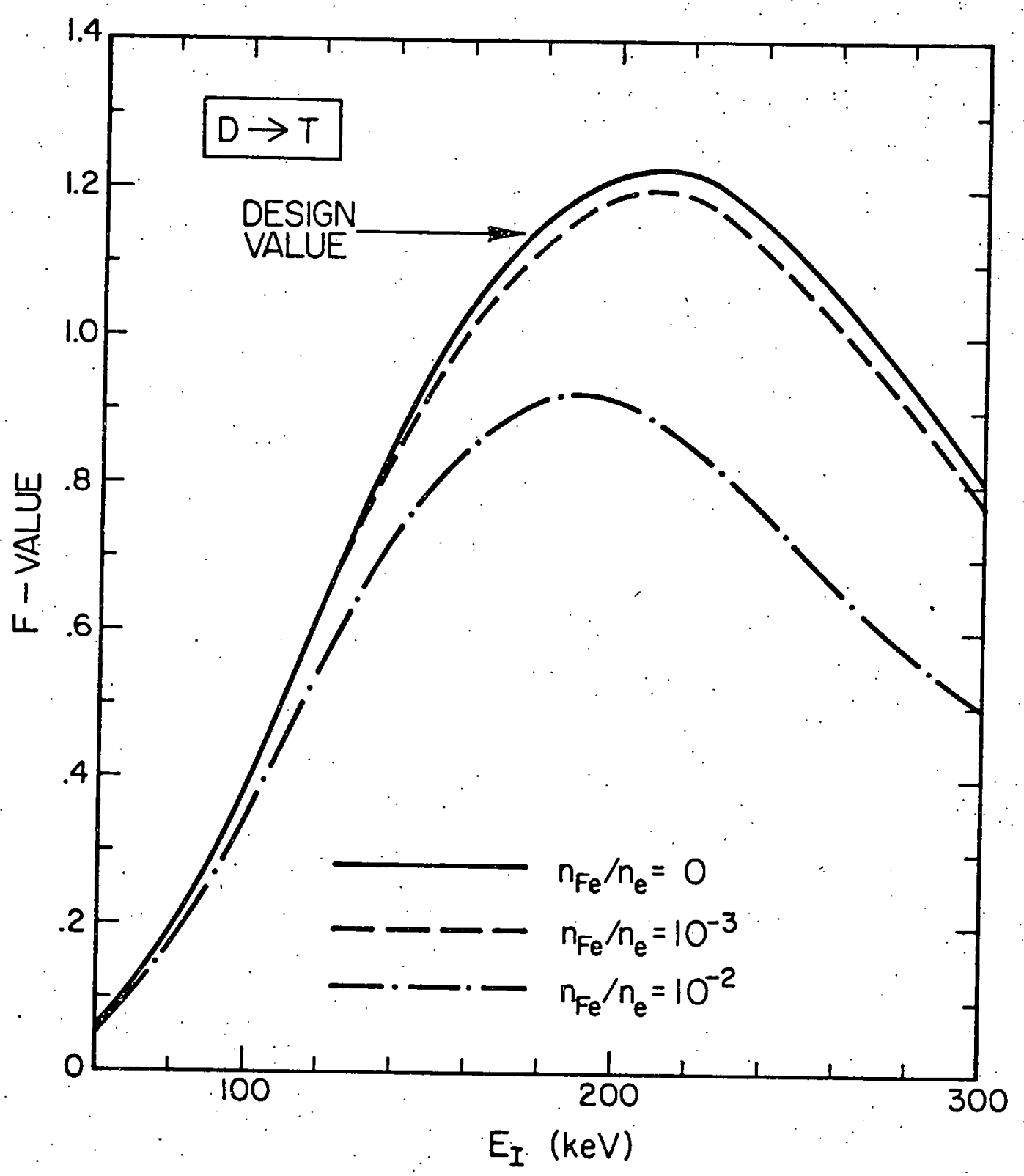

Fig. 3.16 Variation of F-values with different iron impurity concentrations. 
where the sum over $j$ includes all ion species, can then be written $($ for $D \rightarrow T$ ) as:

$$
z_{\text {eff }}=\left(1-26 n_{F e} / n_{e}\right)+\frac{n_{F e}}{n_{e}}(26)^{2} .
$$

For the above concentrations it is found that the effective $Z$ of the plasmas are 7.5 and 1.65 respectively.

For high injection energies it is found that impurities reduce the energy multiplication value by the factor of $n_{i} / n_{e}$. This can be understood by the use of Eqs. (3.4) and (3.5). In this region the drag on the beam ions is mainly due to the background electrons and hence $\langle\mathrm{dE} / \mathrm{dt}\rangle$ is proportional to $\mathrm{n}_{\mathrm{e}}$. However, the fusion power depends upon the number density of the background fusing species (in this case $n_{i}$ ) and hence the $F$-value will vary as $n_{i} / n_{e}$. For low injection energies the drag on the beam ions is mainly due to the background fusing species and hence the F-values for the different concentrations merge into the same value. 
CHAPTER 4

SUMMARY AND CONCLUSION

$\underline{\text { Sensitivity }}$

In the previous chapter the various key parameters $\tau_{E}, \tau_{b}, j_{I}$, $Z_{\text {eff }}$, and $E_{I}$ were varied one at a time to demonstrate trends. Since the TFTR represents a major step forward in plasma physics, it is unclear whether the scaling used to arrive at the "design" values of these parameters is accurate. For this reason, it is important to consider how sensitive the TFTR is to variations in these key parameters. Such a study is frequently referred to as a "sensitivity analysis".

Figure 4.1 summarizes the sensitivity of the $F$-value for $D \rightarrow T$ to changes in each of the key parameters. Here $F_{o}$ is the F-value calculated with the normal values of the design parameters given earlier in Table 3.1 (note that $F_{0}$ has a value of .95 ). The ratio of the perturbed value of a given parameter to its design value is given by $x$. All evaluated points (open circles) in Fig. 4.1 were calculated using the design value for all parameters except for the one which is being varied. The broken straight lines which connect calculated points are included only as a convenience and not to represent the precise variation of the F-values between points.

The results in Fig. 4.1 show that the injection energy $\left(E_{I}\right)$ and injection rate $\left(j_{I}\right)$ are two of the more critical factors in the design. To achieve the relatively high energies desired, the injectors for the TFTR will probably require multistage acceleration. To date, however, there are no working multistage injectors in this country although a special model has been built at Culham. (26-29) Since considerable tech- 


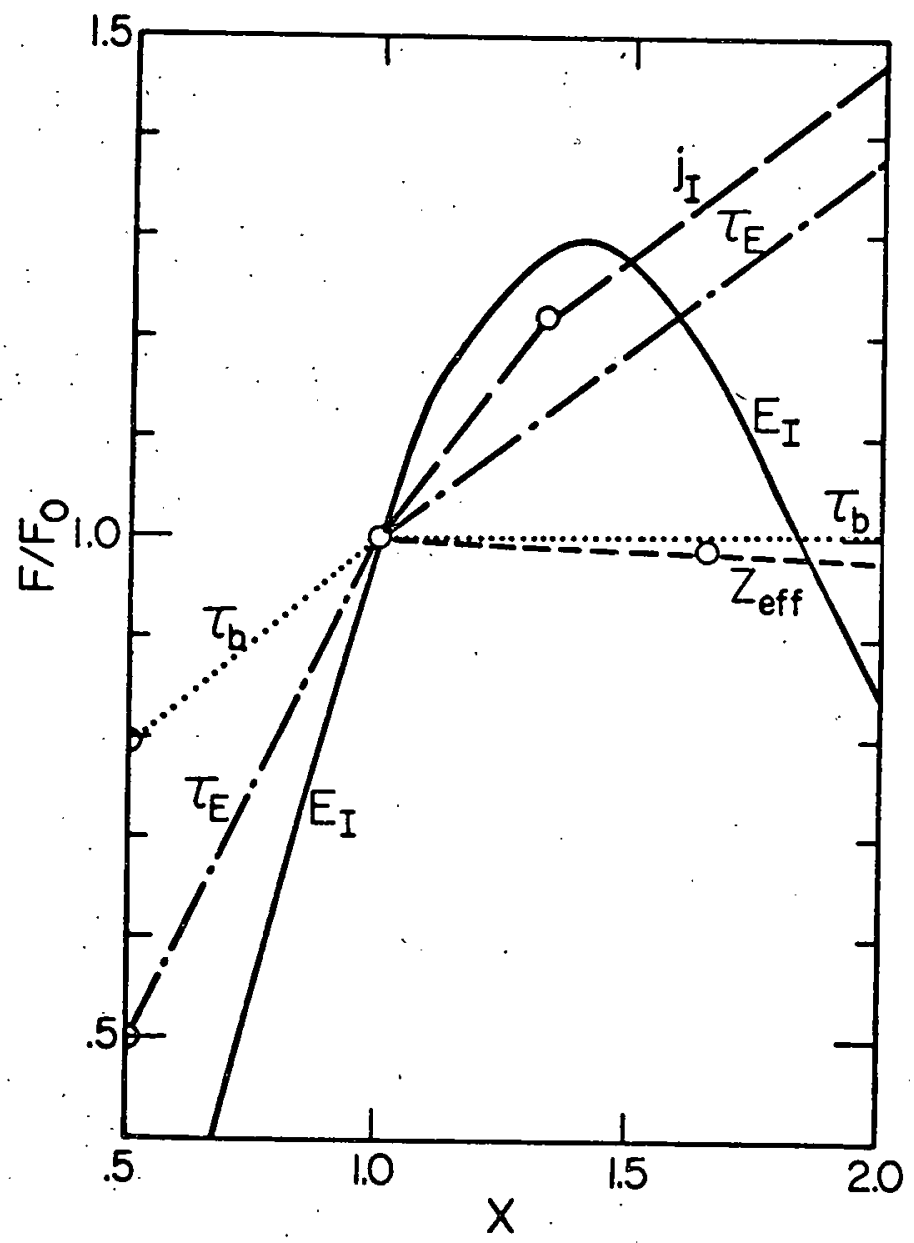

Fig. 4.1 Sensitivity of F-values with respect to the various key parameters: $\tau_{E}, \tau_{b}, j_{I}, z_{\text {eff }}$, and $E_{I}$. 
nological development is required, it is unclear whether the design values for $j_{I}$ and $E_{I}$ will be met. While scaling of the current is reasonably straightforward, voltage breakdown and related problems could limit the energy to levels below the design value. This could cause a serious deterioration in the F-value and hence, the success of TFTR (by obtaining $F=1$ ) is strongly connected to the successful development of multistage injectors.

The F-value is also quite sensitive to the energy confinement time, $\tau_{E}$. It is presently thought that the TFTR plasma will fall into the trapped-ion regime. While this appears as a modest extrapolation of plasma conditions from current devices, it must be remembered that this regime has yet to be obtained experimentally. Thus, achievement of the design value of $\tau_{E}=.2 \mathrm{sec}$ must be viewed with caution. Still, this goal appears to be fairly conservative since, according to Eq. (3.33), a value of $\tau_{E}$ as high as $.43 \mathrm{sec}$ could occur. If this is the case, the F-value would actually increase by as much as a factor of 1.4. Thus, although possible anomalous diffusion or other unexpected losses cannot be completely discounted, satisfactory containment of the target plasma appears to be reasonably assured.

The variation of the F-value with $Z_{\text {eff }}$, shown in Fig. 4.1, is generally negligible for high-Z material such as iron (however, note that this model neglects any line radiation). However, the variation with lower $Z$ impurities (such as hydrogen and oxygen ${ }^{(31)}$ ) may be more significant since for the same $z_{\text {eff }}$ (but with the electron number density, $n_{e}$, at the design value) the number density of the impurity increases with decreasing $Z$ of the impurity (recall Eq. (3.35)). As the impurity density increases, the number density of the fusing background species, $n_{i}$, 
must decrease (recall Eq. (3.34)) . Consequently, for the same $z$ eff, the ratio of $n_{i} / n_{e}$ will decrease with decreasing $z$ of the impurity, leading to lower F-values as discussed in Chapter 3.

As shown in Fig. 4.1, the energy multiplication factor is relatively insensitive to the beam containment time, $\tau_{b}$. Once $\tau_{b}$ exceeds the slowing down time for the injected ions, any further increase has a negligible effect on the F-value. Thus the only problem is if $\tau_{b}$ falls below the slowing down time such that superthermal ions escape prematurely, reducing $F / F_{0}$. However, based on the experience gained with neutral beam heating on the CLEO, ATC, and ORMAK devices, it is thought that the design value of $\tau_{b}=.2 \mathrm{sec}$ represents a realistic prediction.

\section{Conclusion}

The results presented in this work for $\mathrm{D} \rightarrow \mathrm{T}$ operation indicates that the TFTR has an excellent chance of achieving energy breakeven $(F=1)$ if the injectors meet their design criteria. This result is, in fact, consistent with the basic philosophy of the TCT concept. Namely, the use of beam-target fusion reduces the confinement requirements on the target plasma (as opposed to confinement for Maxwellian plasma fusion) but obviously induces strenuous beam requirements. (4) This in effect shifts the stress from magnet technology to injector technology. The TCT experiment was proposed under the premise that the required injector improvements are reasonably assured in a near term schedule.

In addition to the $D \rightarrow T$ results presented here, operation with $D \rightarrow{ }^{3} \mathrm{He}$ has been explored. This is not of interest to achieve energy breakeven, but, as noted in Chapter 2, to provide initial experience with a fusing 
plasma before contamination of the systen with tritium. While the Fvalues for $\mathrm{D}^{3}{ }^{3} \mathrm{He}$ calculated here are quite low, simulation of some characteristics of a $\mathrm{D} \rightarrow \mathrm{T}$ plasma appear to be possible by adjustment of other parameters. For example, a reasonable simulation of $\Gamma$ (beam to background energy ratio) is possible by lowering the background target density. (23). Thus, in this light, $\mathrm{D} \rightarrow{ }^{3} \mathrm{He}$ operation would seem to be the logical step between a non-fusing plasma and a $D \rightarrow T$ plasma.

Finally, it must be stressed that many approximations and assumptions were made to obtain both the $\mathrm{D} \rightarrow \mathrm{T}$ and $\mathrm{D} \rightarrow^{3}$ He results, so that the possibility of modifying these conclusions with further study must be held open. However, the general. trends should be valid and the techniques used should prove useful for future studies.

Recommendations for Future Work ,

In order to refine the results presented in this work, the following ideas might be incorporated in future calculations:

a. Inclusion of the background Maxwellian fusion.

b. Modification of the slowing down model by including the effects of a strong magnetic field and hard collisions.

c. Inclusion of a self-consistent temperature and density profile for the target plasma.

d. Improvement in the treatment of the spatial distribution of energy deposition by the fusion charged products.

e. Expressing the key parameters $\tau_{E}$ and $\tau_{b}$ in terms of the basic plasma parameters of temperature, density, etc.

f. Include other plasma heating mechanisms such as ohmic heating and compression. 
g. Extension of the model to include different ion species (background fusing species, background beam species, and impurity species) with their characteristic temperatures and densities.

h. Extend study to include G-value variation under different compression schemes. 


\section{LIST OF REFERENCES}

1. S. Glasston and R. Loveburg, Controlled Thermonuclear Reactions, (Van Nostrand Reinhold Company, New York, 1960), Chap. '1.

2. Two Component Torus Joint Conceptual Design Study, Vo1. 1-3; Princeton Plasma Physics Laboratory, Princeton, New Jersey and Westinghouse Electric Corporation, Pittsburg, Pennsylvania (1974).

3. W. I. Linlor, Hughes Aircraft Report No. 128, 1959 (unpublished).

4. J. M. Dawson, H. P. Furth, and F. H. Tenney, Phys. Rev. Lett. 26, 1156 (1971).

5. C. Bathke, H. Towner, G. H. Miley, Trans. Am. Nucl. Soc., 17, 41 (1973).

6. H. Towner and G. H. Miley, Trans. Am. Nucl. Soc., 19, 11 (1974).

7. A. A. Husseiny and Z. A. Sabris, Trans. Am. Nuc1. Soc., 17, 43 (1973).

8. J. R. McNally, Jr., Oak Ridge National Report ORNL-TM_3783 (April 1972).

9. R. F. Post, T. K. Fowler, J. Killeen and A. A. Martin, Lawrence Livermore Laboratory Report UCRL-74790 (June 1973).

10. B. H. Duane, "Fusion Cross Section Theory," in Battelle Northwest Laboratory Report BNWL-1685 (1972).

11. N. Jarmie and R. Seagrave, Los Alamos Scientific Laboratory Report LA-2014 (1957).

12. D. L. Jassby, Princeton Plasma Physics Laboratory Report TM-280 (1974).

13. D. V. Sivukhin, Reviews of Plasma Physics, (Edited by M. A. Leontovich, Consultants Bureau, New York 1966), Vol. 4, p. 93.

14. I. P. Shkarofsky, T. W. Johnston, M. P. Bachynski, The Particle $\frac{\text { Kinetics of Plasmas, }}{1966), \text { p. } 261 .}$ (Addison-Wesley Publishing Co., Reading, Mass.,

15. H. P. Furth and D. L. Jassby, Princeton Plasma Physics Laboratory Report, MATT-1040 (January 1974).

16. D. L. Jassby and H. P. Furth, Princeton Plasma Physics Laboratory Report, MATT-1048 (May 1974).

17. R. M. Kulsrud and D. L. Jassby, Princeton Plașma Physics Laboratory Report, MATT-1114 (January 1975). 
18. D. J. Rose and M. Clark, Jx., Plasmas-and Controlled Fusion, (The M.I.T. Press, Cambridge, Mass., 1965).

19. G. Fenske and G. H. Miley, to be published.

20. J. R. McNally, Jr., Nuclear Fusion, 11, 187 (1971).

21. T. Chu, private communication.

22. R. A. Krajcik, Nuclear Fusion, 13, 7.

23. T. W. Petrie and G. H. Miley, University of Illinois Report, COO-2215-27 (March 1975).

24. S. 0. Dean, et al., "Status and Objectives of Tokamak Systems for Fusion Research," USAEC Report WASH-1295 (July 1974).

25. H. L. Berk, et a1., Fifth Conference on Plasma Physics and Controlled Nuclear Fusion Research (IAEA, Tokyo, 1974), paper CN-33/62-3.

26. E. Thompson, "The Ion Optics of a Single Stage Post-Acceleration System," Proc. 2nd Sym. on Ion Sources and Formation of Ion Beams, Berkeley, Calif., pp. II-7-1 to 7-4 (Oct. 1974).

27. D. R. Sweetman, "Neutral Injection Heating," Sym. on Plasma Heating. and Injection, Varenna-Villa Monastero Italy, pp. 117-119 (Oct. 1972).

28. G. W. Hamilton, W. L. Dexter, and B. H. Smith, Lawrence Livermore Laboratory Report UCRL-75547, pp. 5-8 (March 1974).

29. G. H. Miley, Lawrence Livermore Laboratory Report UCRL-51482 (Dec. 1973).

30. R. Conn, W. Houlberg, and J. Kesner, University of Wisconsin Report UWFDM-106 (June 1974). 